\title{
MATCHING STELLAR MODELS TO OSCILLATION DATA
}

\author{
D. B. Guenther and Kevin I. T. Brown \\ Department of Astronomy and Physics, Saint Mary’s University, Halifax, NS B3H 3C3, Canada; guenther@ap.stmarys.ca \\ Received 2003 March 16; accepted 2003 September 5
}

\begin{abstract}
We describe a method to match stellar models to a set of low-l $p$-mode oscillation frequencies. The method provides a quality of fit measure similar to that obtained from a $\chi^{2}$ fit. With a sufficiently dense grid of stellar models, the method can determine the best-fitting model to the oscillation data as constrained solely by the oscillation data. That is to say, the method can be used to constrain the mass, age, composition, surface temperature, and luminosity of a star from the oscillation data. We evaluate the method on solar data and apply the method to the recently observed oscillation data for $\alpha$ Cen and $\alpha \mathrm{UMa}$.
\end{abstract}

Subject headings: stars: individual ( $\alpha$ Centauri A) — stars: interiors — stars: oscillations -

Sun: helioseismology — Sun: interior — Sun: oscillations

\section{INTRODUCTION}

It is our expectation, based on the proven success of helioseismology, that the stellar oscillation observations to be obtained from the proposed satellite missions MOST (Matthews et al. 2000), COROT (Baglin et al. 2002), and Eddington (Favata, Roxburgh, \& Christensen-Dalsgaard 2000) will enable stellar modelers to determine stellar radii and ages, to test modern theories of convection, to study the effects of magnetic cycles and close companion stars, and, in general, to refine the physics of the stellar model. There are, however, new challenges for both the observers and the stellar modelers (Kjeldsen \& Bedding 1995, 2001). Stellar oscillation data are not as well resolved as those for the Sun; both the Sun's brightness and its resolved disk contribute to a high signal-to-noise ratio and the ability to spatially resolve high $l$-value modes. In addition, the mass, age, radius, composition, and luminosity are not as well determined for stars as for the Sun and indeed may have to be constrained by the oscillation data before any significant tests of stellar physics can be undertaken. It has been shown, however, that even at low frequency resolutions, asteroseismic data can be used to constrain the mass and age of single stars of known composition. For stars in binary systems, with known masses, stellar oscillation data can be used to test the mass and distance determinations, and with stellar oscillation data of higher quality, i.e., with frequency resolutions on the order of 1 part in 1000 or better, applied to well-determined stellar systems, one can begin to study the structure of the star's surface layers. Some examples of detailed modeling include: for $\alpha$ Cen, Edmonds et al. (1992), Guenther \& Demarque (2000), Morel et al. (2000), and Thévenin et al. (2002); for Procyon, Barban et al. (1999) and Chaboyer, Demarque, \& Guenther (1999); for $\eta$ Boo, Christensen-Dalsgaard, Bedding, \& Kjeldsen (1995), Guenther \& Demarque (1996), and DiMauro \& Christensen-Dalsgaard (2001); for $\alpha$ UMa, Guenther et al. (2000) and Dziembowski et al. (2001); and for intermediatemass stars in general, Guenther (2002).

To date, our efforts to analyze stellar oscillation data have not been completely satisfactory. Our approach has been rather conventional: (1) several stellar models are constructed that bracket the known observational constraints (typically composition, mass, luminosity, and effective temperature); (2) $p$-mode oscillation frequencies are calculated for the models; and (3) the model spectra are compared with the observed spectrum. Comparisons between the model and observed oscillation spectra, for the most part, are done subjectively, with no quantitative measure of how well the spectra are matched. This method works well for the Sun because even our most basic solar models already reproduce the observed $p$-mode spectrum to within $1 \%$. We believe a different approach is needed for asteroseismology, one that quantifies how well the model spectra match the observations and does not depend on having a close stellar model from which to begin.

Christensen-Dalsgaard (1986) was one of the first astronomers to consider formally how stellar oscillation data might be used to study stars. He proposed characterizing the stellar $p$-mode oscillation spectrum by two parameters, the large and small spacings. With only two parameters to match, the task of comparing models and observed spectra is easier to quantify. In Christensen-Dalsgaard's approach, the distribution of oscillation frequencies as a function of radial order $n$ and azimuthal degree $l$ are assumed to closely fit the asymptotic formula (Tassoul 1980):

$$
\nu_{n l} \simeq\left(n+\frac{l}{2}+\frac{1}{4}+\beta\right) \Delta \nu-\left(A L^{2}-\epsilon\right) \frac{\Delta \nu^{2}}{\nu_{n l}},
$$

where $R$ is the radius of the star and $L \equiv l+\frac{1}{2}$. The characteristic spacing $\Delta \nu$ is related to the run of sound speed $c$ by

$$
\Delta \nu=\left(2 \int_{0}^{R} \frac{d r}{c}\right)^{-1}
$$

and $A$ is related to the run of sound speed by

$$
A=\frac{1}{4 \pi^{2} \Delta \nu}\left(\kappa-\int_{0}^{R} \frac{d c}{d r} \frac{d r}{r}\right)
$$

The constants $\beta, \epsilon$, and $\kappa$ depend primarily on the structure of the star near the surface. The formulae reveal that modes adjacent in $n$ are separated in frequency by approximately $\Delta \nu$ :

$$
\Delta_{n, l} \equiv \nu_{n, l}-\nu_{n-1, l} \propto \Delta \nu .
$$


Modes adjacent in $l$ are separated by approximately $\Delta \nu / 2$. If the spacings were exactly linear, then the frequencies of modes of higher degree, e.g., $l=2$ and 3 , would coincide with the frequencies of lower degree modes, e.g., $l=0$ and 1 , respectively. They do not, and the difference is called the "small spacing," which is related to the asymptotic formula by

$$
\delta_{n, l} \equiv \nu_{n, l}-\nu_{n-1, l+2} \propto \Delta \nu \int_{0}^{R} \frac{d c}{d r} \frac{d r}{r} .
$$

The large spacings are sensitive to the sound speed in the outer layers, and the small spacings are sensitive to the slope of the sound speed in the interior.

By comparing the average large and small spacings of a stellar oscillation spectrum with the same spacings determined from models, one can constrain the radius and age of a star, assuming the composition is already well determined. The large and small spacings are especially useful when the oscillation data are not well determined. For the higher quality data expected from satellites, we desire a method that utilizes all the diagnostic information contained in the data. Brown et al. (1994) consider a more general method that utilizes all the observational constraints of a star or binary system. Their method, based on singular value decomposition, uses the oscillation data to reduce the overall error of all the observational uncertainties. Models are calculated for the star being investigated. The parameters of the model are varied so that derivatives of the parameters can be determined to first order. With these variations quantified, they use a $\chi^{2}$ minimization procedure to determine the optimum parameters that fit the data. The method requires the reference models to be close enough to the actual star that the perturbations to the parameters required to fit the star are small.

As an aside, we note that inversion techniques have been successful in probing the interior of the Sun, e.g., inversion methods are responsible for determining the depth of the convection zone (Christensen-Dalsgaard, Gough, \& Thompson 1991; Basu 1997), the interior rotation rate (Schou et al. 2002), and the helium abundance in the convection zone (Dziembowski, Pamiatnykh, \& Sienkiewicz 1991). They will, however, be more difficult to use on stellar oscillation data. The number of modes available to form the kernels, used to span the interior of the star, is limited to the low- $l$ modes, making it more difficult to isolate distinct depths in the star. Perhaps more significantly, the reference stellar model on which the inversions are based is unlikely to be as close to the actual star as our solar models are to the Sun, and therefore there is an increased likelihood that the inversions will not converge to the correct solution. Regardless, we do expect that inversion techniques will be developed for stars that explore specific features in the interior, such as convection zone depth. As we propose in the conclusions section of this paper, inversion techniques can be used in conjunction with the method described in this paper.

We propose a direct method that, although computationally laborious to set up, makes use of all of the $p$-mode oscillation frequencies and provides a quantitative measure of how well the oscillation data fit the model. The method, described in this paper, relies on the computation of oscillation spectra for a vast and dense grid of stellar models. The observed stellar oscillation spectrum is compared one by one with the model spectra, and the closest match (or matches) is tabulated. The match itself is quantified by a single parameter, $\chi^{2}$. Once the models have been constrained by the oscillations, other observational data can be easily folded in to further constrain the models. By constraining the models first with only the observed oscillation spectrum, we are able to quantify the quality of fit between the observed and model spectra. Our method, through brute force, allows the stellar modeler to greatly reduce the parameter space of possible models to those consistent with the oscillation data. The resulting models, which are probably very close in parameter space to the target star, can then be used as a starting point in the Brown et al. (1994) approach. The Brown et al. approach would then be used to extend the analysis to a wider variety of parameters.

In the following sections of this paper we describe the mode-matching method in detail. We present results of tests on low- $l$ solar data, and we describe successful applications of the mode-matching method to the recently observed $p$-mode oscillation modes on $\alpha$ Cen A by Bouchy \& Carrier (2002) and on $\alpha$ UMa by Buzasi et al. (2000).

\section{MODE-MATCHING METHOD}

\subsection{Overview}

In this section we describe a procedure that allows us to match stellar models to oscillation data. We are able to determine the stellar model or range of models whose oscillation spectra most closely match the given oscillation spectrum, and we are able to quantify the match.

The method is relatively straightforward. We search through an extensive grid of stellar models and find the model or models whose oscillation spectra most closely match the oscillation data. A $\chi^{2}$ formulation is used to quantify how well the given oscillation spectrum matches the model spectra. Models in the grid are parameterized by mass, age, $Z$ (metal abundance), $Y$ (helium abundance), and whether or not $Y$ and $Z$ diffusion are included in the model physics. The number of models, i.e., the density of the grid, is set by the need to be able to follow small changes in frequencies of individual modes as one moves from one model to the next along a single parameter axis in the grid.

\subsection{The Grid}

All stellar model sequences are calculated using the Yale stellar evolution code (YREC; Guenther et al. 1992). For opacities, the OPAL98 tables (Iglesias \& Rogers 1996) are used for temperatures above $6000 \mathrm{~K}$, and the Alexander \& Ferguson (1994) "molecular" opacity tables are used below $15,000 \mathrm{~K}$; for the equation of state, the tables from Lawrence Livermore National Laboratory (Rogers 1986; Rogers, Swenson, \& Iglesias 1996) are used. In the region of temperature overlap, a linear ramp function is used to smooth the transition between the two opacity tables. The nuclear energy generation routines are based on the latest cross sections (Bahcall, Pinsonneault, \& Basu 2001).

All stellar evolutionary tracks begin on the zero-age main sequence (ZAMS) and end near the base of the giant branch or at $15 \mathrm{Gyr}$ for the lower mass tracks. The ZAMS starting models themselves were obtained by evolving Lane-Emden gas spheres (Chandrasekhar 1957, pp. 273-274) from the Hayashi track to the ZAMS using YREC. The stellar models have approximately 2000 shells, with one-third of the shells covering the interior, one-third covering the outer envelope, which encompasses less than $1 \%$ of the total mass, and onethird covering the atmosphere. The atmosphere is a gray atmosphere in the Eddington approximation. 
The mixing-length parameter $\alpha$ was obtained from a calibrated solar model constructed using the same physics, i.e., opacities, equation of state, and atmosphere, as used in the stellar models. Convective overshoot and rotation are not included in the models. It would be desirable to extend the parameter space of the grid to include $\alpha$, but at this time the computations would take too long to complete (over a year). In the future we will isolate a small section of the grid and extend it with a range of $\alpha$ to test whether the oscillation data can constrain the four parameters, mixing length, composition, age, and mass, without degeneracy.

Evolutionary tracks were constructed for masses within the range $0.71-5.00 M_{\odot}$. A variety of helium and metal abundances were considered, specifically, $(Y, Z)=(0.27,0.01)$, $(0.27,0.02),(0.27,0.04),(0.26,0.02)$, and $(0.25,0.02)$. The relative mixture of heavier than helium elements is solar, as summarized by Grevesse, Noels, \& Sauval (1996). We have also calculated evolutionary tracks at $(Y, Z)=(0.27,0.02)$ and $(0.27,0.04)$, for which the effects of gravitational settling (i.e., diffusion) of helium and metals are included in the modeling physics. The diffusion formulation is identical to that described by Bahcall, Pinsonneault, \& Wasserburg (1995). Table 1 summarizes the parameter range of the grid. The grid's resolution is high in mass and age but not in composition. Over the next few years, both the mass range and the composition resolution will be increased for future applications.

Although we describe our parameterization of the grid in terms of mass and age (and composition), the age dimension is actually delineated by the arc length $\lambda$, measured from the ZAMS, that a model moves along its evolutionary track in an $\mathrm{H}-\mathrm{R}$ diagram. The correspondence between age and structural change within the star is nonlinear. The rate of evolution, i.e., the rate at which the internal structure changes, varies significantly with the phase of evolution, e.g., it is slow on the main-sequence and fast on the giant branch. Furthermore, the rate evolution varies significantly with the mass, being proportional to approximately the fourth power of the mass (on the main sequence). Equally spaced models along the track in $\lambda$ more closely correspond to equal-sized changes in the frequencies of the models. Therefore, along the age dimension of the grid, we select approximately 150 models, for $1 M_{\odot}$, that are, in fact, equally spaced in $\lambda$.

We found during initial experimentation that the grid resolution in mass and age had to be relatively high in order to follow smoothly the variations in $\chi^{2}$ as a function of mass and age. Our grid needs to have a mass resolution of $0.002 M_{\odot}$; that is, adjacent models in our grid must be separated by no more than $0.002 M_{\odot}$. Along the age axis, our grid needs to have the models spaced closely enough in time that for a

TABLE 1

Grid Parameters

\begin{tabular}{cccc}
\hline \hline & \multicolumn{3}{c}{ Mass Range } \\
$\left(M_{\odot}\right)$ & Diffusion $Y$ and $Z$ \\
\hline $0.01 \ldots \ldots$ & 0.27 & $0.71-1.30$ & No \\
$0.02 \ldots \ldots$ & 0.25 & $0.91-1.10$ & No \\
$0.02 \ldots \ldots$ & 0.26 & $0.91-1.10$ & No \\
$0.02 \ldots \ldots$ & 0.27 & $0.51-5.00$ & No \\
$0.02 \ldots \ldots$ & 0.27 & $0.81-1.30$ & Yes \\
$0.04 \ldots \ldots$ & 0.27 & $0.80-1.30$ & No \\
$0.04 \ldots .$. & 0.27 & $0.81-1.30$ & Yes \\
\hline
\end{tabular}

$1 M_{\odot}$ model we produce approximately 2000 distinct models along its evolutionary track from the ZAMS to the base of the giant branch. If we were to adopt this grid density, then the total number of models would exceed approximately 1 million in our $Y=0.27, Z=0.02$ diffusion portion of the grid alone. With our current computational resources, it would take over a year to compute the oscillation spectra. Instead of directly computing all these models and their oscillation spectra, we constructed a lower resolution grid in mass and $\lambda$ and then linearly interpolated between the models. The grid resolution requirements in mass and $\lambda$ are, as a consequence, each almost an order of magnitude lower. Specifically, the tracks are separated in mass by $0.01 M_{\odot}$ $\left(0.02 M_{\odot}\right.$ for masses greater than $\left.2.0 M_{\odot}\right)$, and along each track, equally spaced in $\lambda$, we extract from 40 models at $0.71 M_{\odot}$ to 170 models at $1.4 M_{\odot}$.

The interpolation procedure itself works by first identifying the four models, $\left(M_{i}, \lambda_{j}\right),\left(M_{i+1}, \lambda_{j}\right),\left(M_{i}, \lambda_{j+1}\right)$, and $\left(M_{i+1}\right.$, $\left.\lambda_{j+1}\right)$, that surround the $(M, \lambda)$ values that parameterize the model spectra being sought. The $p$-modes in each of the four surrounding models are then matched to each other. As long as the grid resolution is high enough that the mode frequencies have not changed by too much $(\sim \Delta \nu / 4)$, the corresponding modes in the adjacent models can be determined unambiguously. Once the frequencies of the four models have been matched up, two-dimensional interpolation is carried out to produce an interpolated oscillation spectrum at $(M, \lambda)$.

The models were input into our nonradial, nonadiabatic stellar pulsation code (Guenther 1994), and $l=0,1,2$, and 3 $p$-mode frequencies from $n=3$ to 35 were calculated. Both adiabatic and nonadiabatic (radiative gains and losses) frequencies were computed. In this study, we focus on the $l=0$ to 2 nonadiabatic $p$-mode frequencies.

$$
\text { 2.3. } \chi^{2}
$$

We utilize a $\chi^{2}$ formulation to quantify how well two oscillation spectra match each other. When comparing an observed oscillation spectrum with the spectra in our grid, we expect the minima in $\chi^{2}$ to occur in regions of the grid, as parameterized by mass, age, and composition, where the observed oscillation spectrum most closely matches the model spectra. We define our $\chi^{2}$ by

$$
\chi^{2} \equiv \frac{1}{N} \sum_{i=1}^{N} \frac{\left(\nu_{\mathrm{obs}, i}-\nu_{\mathrm{mod}, i}\right)^{2}}{\sigma_{\mathrm{obs}, i}^{2}+\sigma_{\mathrm{mod}, i}^{2}},
$$

where $\nu_{\mathrm{obs}, i}$ is the observed frequency for the $i$ th mode, $\nu_{\bmod , i}$ is the corresponding matched model frequency for the $i$ th mode, $\sigma_{\mathrm{obs}, i}$ is the observational uncertainty for the $i$ th mode, $\sigma_{\text {mod, } i}$ is the model uncertainty for the $i$ th mode, and $N$ is the total number of matched modes.

We estimate our stellar model uncertainties $\left(\sigma_{\bmod }\right)$ by scaling the solar model uncertainties as determined by comparing the low- $l p$-mode frequencies calculated from our standard solar model with solar oscillation data. Figure 1 shows the relative frequency differences $f_{n}$ between our standard solar model frequencies ( $\left.\nu_{\text {nonad }}\right)$ and the Sun's frequencies $\left(\nu_{\odot}\right)$ plotted against radial order $n$. The solar data are from GONG (Global Oscillation Network Group; Harvey et al. 1996; see $\S 3.1$ ). The frequency scale along the top $x$-axis (taken from the $l=1 p$-modes) is an approximate match to $n$ for low- $l$ solar $p$-modes. As seen in Figure 1, our standard solar model $p$-mode frequencies (from $l=0$ to 3 ) match the 


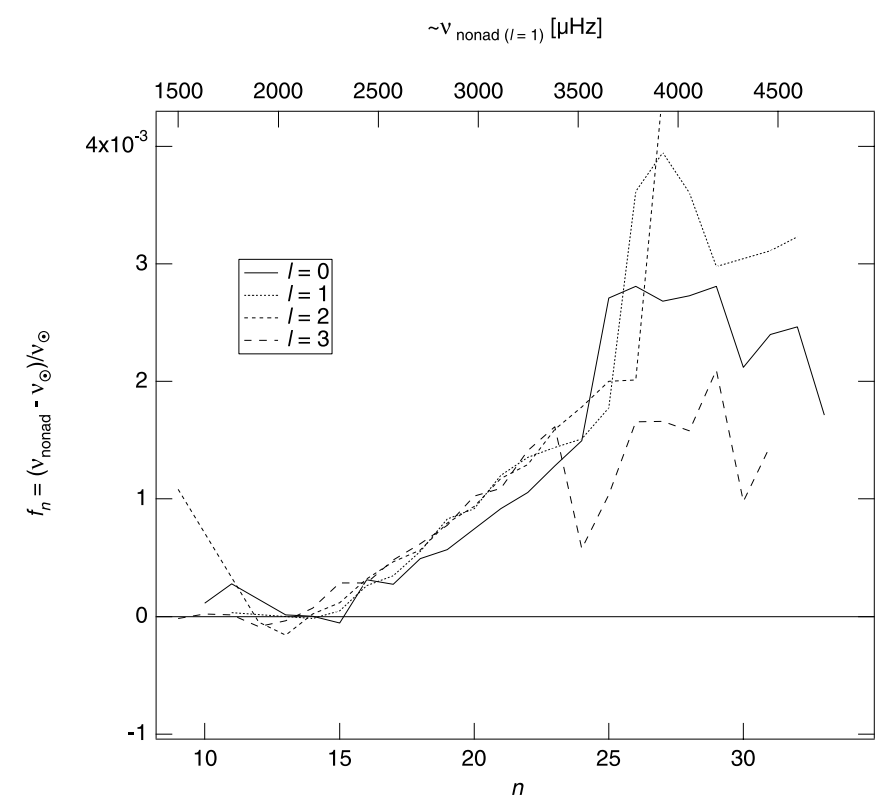

FIG. 1.-Uncertainties in the model spectra determined from a calibrated solar model. The relative frequency differences, model minus Sun, as a function of $n$ for $l=0,1,2$, and $3 p$-modes are shown. The values $f_{n}$ (obtained from a fit to the average relative frequency differences for the Sun) are used in the mode-matching software to calculate the model uncertainties. Along the top $x$-axis, the frequency scale approximately matches the $n$-values.

solar frequencies to better than $0.3 \%$. The fit is best $(\sim 0.01 \%)$ for the low- $n$ modes. The systematic growth in error at higher frequencies (higher $n$ ) is associated with the inherent uncertainties in the modeling of the superadiabatic layer of the Sun via the mixing-length theory. Li et al. (2002) have shown that much of this discrepancy can be eliminated by incorporating the results of numerical stellar convection in both the model and the $p$-mode frequency calculation (see also Rosenthal et al. 1999; Robinson et al. 2003).

We assume that the stellar model frequency uncertainties are similar to the discrepancies found between our solar model frequencies and the observed frequencies. We use the solar frequency discrepancy $f_{n} \equiv\left(\nu_{\text {nonad }, n}-\nu_{\odot, n}\right) / \nu_{\odot, n}$ to compute the stellar model frequency uncertainty; that is, we compute $\sigma_{\text {mod, } n}$ using $\sigma_{\bmod , n}=f_{n} \nu_{n}$, where again $n$ refers to the radial order of the mode. Table 2 lists the values of $f_{n}$ that we use here, as estimated from Figure 1. We do not correct our stellar model frequency determinations for the systematic shift in frequencies at higher $n$; hence, as a consequence, we expect a systematic error in our results. If we were to correct for the systematic shift in frequencies, for example, by fitting a polynomial to the differences and then applying this fit as a correction to the frequencies, we would be able to reduce the model uncertainty $\sigma_{\bmod , n}$ at higher $n$. This, in some sense, would be equivalent to correcting for the model errors in the surface layers.

If the observational and model spectra lie within the uncertainties of the observations and the uncertainties in the model, then our weighted $\chi^{2}$ will be less than or equal to 1 . Because the errors in the oscillation frequencies are not independent, we cannot directly interpret a $1 \sigma$ variation in $\chi^{2}$ as a $1 \sigma$ uncertainty in our mass, age, and composition parameter space. In order to determine the true $1 \sigma$ uncertainty range in parameter space, we will have to carry out a Monte Carlo simulation of the parameters within our grid.
TABLE 2

Model Uncertainties

\begin{tabular}{|c|c|c|c|}
\hline$n$ & $f_{n}$ & $n$ & $f_{n}$ \\
\hline $1 \ldots$ & 0.00008 & $21 \ldots \ldots$ & 0.0011 \\
\hline $2 \ldots \ldots$. & 0.00008 & $22 \ldots$ & 0.0013 \\
\hline $3 \ldots$ & 0.00008 & $23 \ldots \ldots$ & 0.0015 \\
\hline $4 \ldots \ldots$. & 0.00008 & $24 \ldots \ldots$ & 0.0018 \\
\hline $5 \ldots \ldots$ & 0.00008 & $25 \ldots \ldots$ & 0.0021 \\
\hline $6 \ldots \ldots$ & 0.00008 & $26 \ldots \ldots$ & 0.0024 \\
\hline $7 \ldots \ldots$. & 0.00008 & $27 \ldots \ldots$ & 0.0026 \\
\hline $8 \ldots \ldots$. & 0.00008 & $28 \ldots \ldots$ & 0.0028 \\
\hline $9 \ldots \ldots$. & 0.00008 & $29 \ldots \ldots$ & 0.0029 \\
\hline $10 \ldots \ldots$ & 0.00008 & $30 \ldots \ldots$ & 0.0029 \\
\hline $11 \ldots .$. & 0.00008 & $31 \ldots \ldots$ & 0.003 \\
\hline $12 \ldots \ldots$ & 0.00008 & $32 \ldots \ldots$ & 0.003 \\
\hline $13 \ldots \ldots$ & 0.00008 & $33 \ldots \ldots$ & 0.003 \\
\hline $14 \ldots \ldots$ & 0.0001 & $34 \ldots \ldots$ & 0.003 \\
\hline $15 \ldots \ldots$ & 0.0002 & $35 \ldots \ldots$ & 0.003 \\
\hline $16 \ldots \ldots$ & 0.0003 & $36 \ldots \ldots$ & 0.003 \\
\hline $17 \ldots \ldots$ & 0.0004 & $37 \ldots \ldots$ & 0.003 \\
\hline $18 \ldots \ldots$ & 0.0006 & $38 \ldots \ldots$ & 0.003 \\
\hline $19 \ldots \ldots$ & 0.0008 & $39 \ldots \ldots$ & 0.003 \\
\hline $20 \ldots \ldots$ & 0.0009 & $40 \ldots \ldots$ & 0.003 \\
\hline
\end{tabular}

\subsection{Mode Matching}

We are given a monotonically increasing list of frequencies, the observed oscillation spectrum, and a table, in $n$ and $l$, of model frequencies and are asked to find the best match between the two. In general, neither the $n$ - nor the $l$-values of the observed modes are known. The degree $l$ of the observed oscillations can be inferred by plotting the frequencies in an echelle-style diagram (see, for example, Fig. 8 in Bouchy \& Carrier 2002), but ambiguities can arise around which modes are of even degree and which modes are of odd degree. Through trial and error we found the following direct approach of matching an observed spectrum and a model spectrum to work the best.

If we assume that the degree $l$ of the mode is known, then the list of observed frequencies are rearranged so that the list begins first with $l=0$ modes, then $l=1$ modes, and then $l=2$ modes. The modes for each degree $l$ are listed in order of increasing frequency. With this ordering, each mode in the observed frequency list is compared one by one with the model frequencies with the same $l$. We note that if the uncertainties in the observed frequencies are less than the small spacing, as will always be the case if the $l$-values can be identified, then it does not matter if the observed frequencies are listed by $l$-value and the search is restricted to matching $l$-values. Indeed, we have confirmed that listing all the modes in order of increasing frequency, regardless of $l$, and searching through all $l$-values produces identical results.

The closest model frequency to the first observed frequency is identified and marked as being selected. The closest model frequency to the next observed frequency is then identified. The search for the closest model frequency is restricted to model frequencies that have not already been selected. The process continues through all observed frequencies. Once all the observed modes have been matched to unique model modes, $\chi^{2}$ is computed.

The matching method clearly gives the observed lower frequencies priority. At first thought, one might suspect that the method will fail to find the best match in all cases, since we can imagine a case in which the first frequency $\left(\nu_{\mathrm{obs}, 1}\right)$ in 
the observed frequency list is matched to a model frequency that, in fact, more closely matches an observed frequency farther down the list $\left(\nu_{\mathrm{obs}, i}\right)$. One is confronted with the frightening thought that in order to ensure that the optimum match is being used to calculate $\chi^{2}$, the searching process should be repeated for all possible permutations of ordering in the observed frequencies. Fortunately, this is not necessary, because the observed and model frequencies have small uncertainties and are relatively evenly spaced. If, in the above example, we allowed the closest matching frequency to go first $\left(\nu_{\mathrm{obs}, i}\right)$, we would still have to find a match to the first frequency $\left(\nu_{\mathrm{obs}, 1}\right)$. In both cases, one mode is going to be poorly matched. When the observed modes do not match well, then the ordering does not matter, since a high $\chi^{2}$ value will be obtained regardless, and if the modes do match well, then each mode will be correctly matched. If the $l$-values of the observed modes are unknown and if the observational uncertainties are comparable to the small spacing, then ambiguities in matching the $l=0$ and $l=2$ modes will occur. However, this is a true reflection of the quality of the observations, and one cannot expect the mode-matching algorithm to find better matches (outside random chance) than the uncertainties in the data allow.

To improve the efficiency of the mode-matching algorithm, we restrict the search in model frequencies to modes that are within $\Delta \nu_{\text {mod }} /$ NSPACE of the observed mode's frequency. If no mode is found, the observed mode is marked as unmatched. If more than NREJECT modes are unmatched, then the model is rejected and no $\chi^{2}$ is calculated. NSPACE and NREJECT are integers that can be adjusted by the user of the software. Unless stated otherwise, the results reported here use NSPACE $=4$ and NREJECT $=5$. The software outputs the number of matched frequencies for each $\chi^{2}$ determination, so it is easy to identify $\chi^{2}$ values computed with fewer than the total number of input frequencies. In practice, we found that the $\chi^{2}$ curves have poorly defined minima when one or more modes are off by more than $10 \%$ of $\Delta \nu_{\bmod }$.

We have assumed that the $l$-values of the observed modes are known for our tests on the Sun and $\alpha \mathrm{UMa}$ (radial modes) and are unknown for our tests on $\alpha$ Cen A. The software has been written so that the user can choose to use or not use the $l$-values when searching the model modes.

\subsection{Composition}

The grid so far constructed does not encompass a large range in helium or metal abundances, nor is the resolution in the few compositions sampled high enough to enable us to smoothly follow changes in $\chi^{2}$ along the helium and metal abundance dimensions of the grid. Regardless, we are able to show that the weighted $\chi^{2}$ is sensitive to composition.

\subsection{Analysis Strategy}

Because $p$-modes are regularly spaced, the code will either match-up nearly all of the modes when the large spacing of the model is close to the large spacing of the observed modes or match-up few, if any, modes when the large spacing is not close. The basic strategy when analyzing stellar oscillation data for the first time involves some trial and error. We begin by studying the $\chi^{2}$ results. If they do not show well-defined minima as a function of age, we then examine the mode matches themselves, looking for isolated modes that are consistently being poorly matched when the other modes are well matched. If we think we have a "bad" mode, we can either remove the bad mode from the data, setting it aside for further scrutiny later, or increase NSPACE so that the rejection tolerance is tight enough to reject the bad mode automatically.

We have found in our own trials that a single bad frequency, especially if it is a low frequency at which the model uncertainties are low, can skew the mode matches so that other modes in the frequency vicinity also appear to be poorly matched. Needless to say, one should be conservative when rejecting modes.

\section{7. $\chi^{2}$ Plots}

$\chi^{2}$ values are calculated for every model (a function of mass, age, and composition) in the grid. When $\chi^{2}$ values for a single composition are plotted as a function of mass and age, we find that the lowest values of $\chi^{2}$ lie very close to a curved two-dimensional surface. As an example, we show in Figures $2 a$ and $2 b$ the $\chi^{2}$ results for fits to $\alpha$ Cen A's observational data (to be discussed in $\S 4$ ). Clearly, for $\chi^{2}<5$ (the bifurcation of the surface above $\chi^{2}=8$ is discussed in $\S 4)$, the corresponding models have oscillation spectra that are close to the observed spectrum. When the models are constrained to have oscillation spectra that closely match the observed spectrum, the values of mass and age are constrained such that mass and age are directly correlated. As a consequence, we can simplify the presentation and analysis of our results by showing projections of this two-dimensional surface on the $\chi^{2}$ versus mass plane and the $\chi^{2}$ versus age plane. The $\chi^{2}$ versus mass plane and the $\chi^{2}$ versus age plane plots are not slices through the data. They show all of the $\chi^{2}$ values calculated within the range of the axes. The minimum value of $\chi^{2}$ in a $\chi^{2}$ versus mass plot corresponds to the same model as the minimum value of $\chi^{2}$ in a $\chi^{2}$ versus age plot. Figures $2 a$ and $2 b$ are discussed further in $\S 4$ when we interpret the $\chi^{2}$ results for $\alpha$ Cen A.

\section{TESTS}

\subsection{Solar Oscillations}

We begin our testing with the Sun. Models of the Sun and the observations are well established. The solar $p$-mode frequencies are determined to better than 1 part in 10,000. The Sun's mass, luminosity, and radius are known to $0.1 \%$, the age to $1 \%$, and the composition to $10 \%$ (Guenther \& Demarque 1997). The frequencies of the $p$-mode spectrum of the standard solar model, a model that is constrained by the observed nonoscillation constraints of age, mass, luminosity, radius, and composition, match the Sun's $p$-mode frequencies to within $0.3 \%$.

To evaluate the mode-matching software, we use the first month of GONG data (Harvey et al. 1996) for 2001, specifically, the $m=0$ values from the mrvlf010104d000, mrv1f010104d001, and mrv1f010104d002 data packages. ${ }^{1}$ We also use the BiSON data (Birmingham Solar Oscillation Network; Chaplin et al. 1999), and the SOHO/VIRGO data (Solar and Heliospheric Observatory/Variability of Irradiance and Gravity Oscillations; Appourchaux et al. 1998). The GONG and BiSON data are derived from ground-based networked telescopes using spectral line Doppler shift measurements, and the $\mathrm{SOHO}$ /VIRGO data are obtained from the Luminosity Oscillations Imager, which measures intensity

\footnotetext{
${ }^{1}$ With regard to the $\chi^{2}$ curves shown here, the difference between using $m=0$ values and using $m$-averaged values was found to be insignificant.
} 


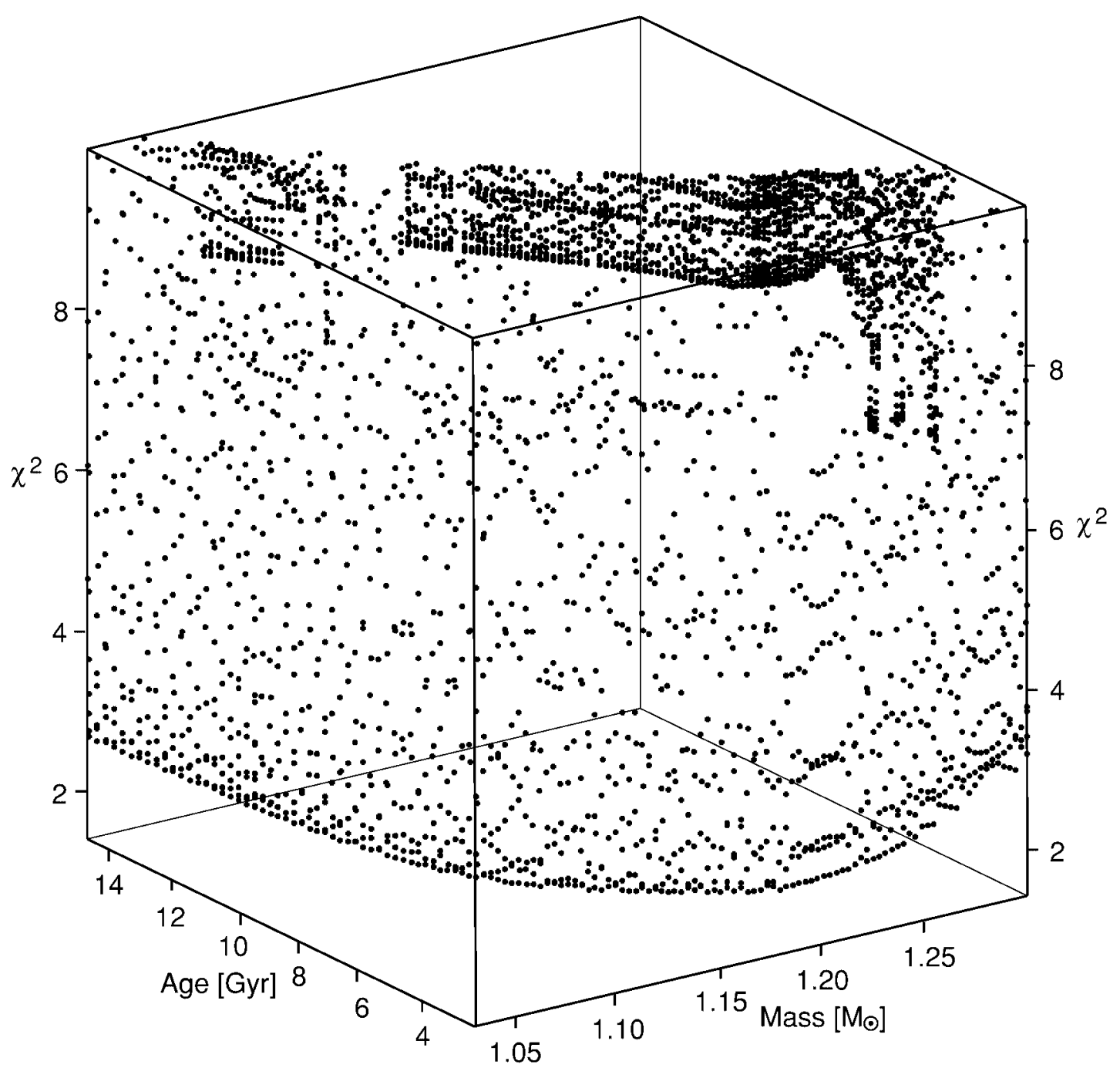

FIG. $2 a$

FIG. 2. - $\chi^{2}$ values calculated for $\alpha$ Cen A data using the $Z=0.04, Y=0.27$ diffusion portion of the model grid, plotted as a function of age and mass. Panels $a$ and $b$ show different orientations of the same data to better reveal the curved two-dimensional surface within which most of the best-fitting models $\left(\chi^{2}<10\right)$ lie. A general discussion of these figures appears in $\S 2.7$. A detailed discussion within the context of interpreting $\alpha$ Cen A data appears in $\S 4$.

variations, on board the $\mathrm{SOHO}$ satellite. We use only the $l=0,1$, and $2 p$-mode frequencies. The range in frequency and $n$ for each data set is listed in Table 3.

First considering the BiSON data, Figure 3 shows the $\chi^{2}$ values plotted against age for the models in the $Y=0.27, Z=$ 0.02 diffusion portion of the grid, and Figure 4 shows the same $\chi^{2}$ values plotted against mass. Figures 3 and 4 are projections of $\chi^{2}$ versus mass and age onto the $\chi^{2}$ versus age and $\chi^{2}$ versus mass planes, respectively. In Figure $4 \chi^{2}$ values only exist for masses greater than $0.85 M_{\odot}$ and less than $1.13 M_{\odot}$ because the spectra of the models outside this mass range do not meet the minimum criteria for a match (see $\S 2.4$ ).

A well-defined minimum in $\chi^{2}$ is visible in both Figures 3 and 4. The minimum in $\chi^{2}$ as a function of age is near the solar age, 4.55 Gyr (Guenther 1989), and the minimum in $\chi^{2}$ as a function of mass is near $1 M_{\odot}$. In other words, there is a single well-defined model from the models shown $(Y=0.27$, $Z=0.02$, diffusion) that best matches the Sun's $l=0,1$, and $2 p$-mode spectrum. The Sun's mass and age have been constrained exclusively by the modeling physics and the oscillation data.
In Figure 5 we show a zoomed-in view from Figure 4, with lines connecting $\chi^{2}$ values of models with the same stellar mass and evolving age. Each curve that dips downward corresponds to a different mass. The masses, in units of $M_{\odot}$, of several of the curves are labeled. Points along each curve correspond to evolving models for which $\chi^{\odot}$ values are calculated. Along a given mass track, the $p$-mode spectra of the models for the most part do not match the Sun's spectrum. At some point, however, the model spectrum comes close enough to the observed spectrum that $\chi^{\odot}$ can be calculated. Initially, the frequencies do not match well, and the $\chi^{\odot}$ values are very high $(>1000)$. As the model evolves and the oscillation frequencies squeeze more closely together, there is a point at which the model and observed spectrum match up the best before growing apart again. In Figure 5 the point of closest match between the model and observed spectra for a given mass corresponds to the minimum on each mass track. The minimum value of $\chi^{2}$ for each mass varies with mass. In Figure 3 the bottom edge of the $\chi^{2}$ values, which corresponds to the minimum $\chi^{2}$ value for each mass track, defines a relatively smooth curve. Because the minimum for each curve 


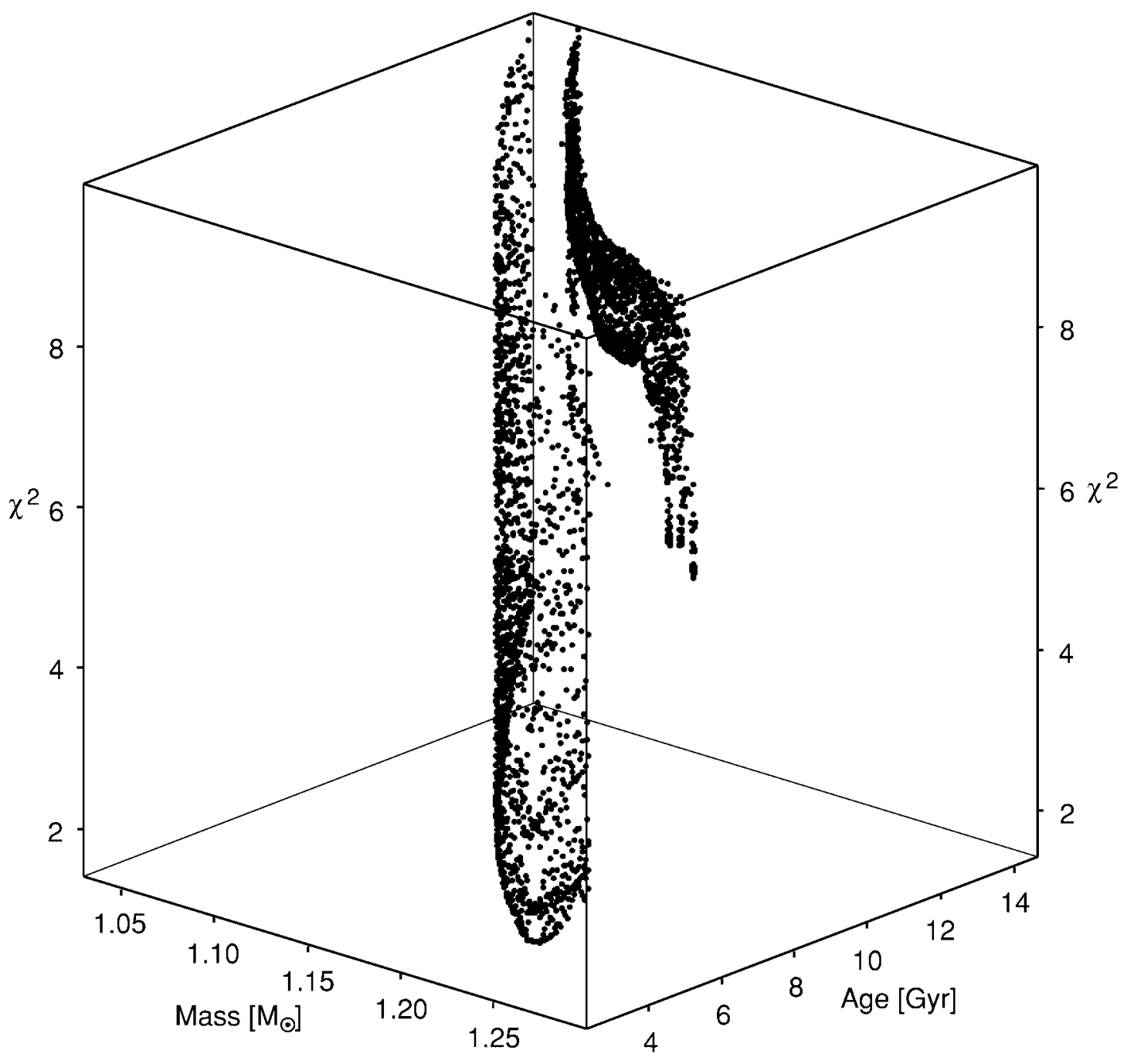

FIG. $2 b$

is selected from three to four points that span the region of the minimum, the minimum value of $\chi^{2}$ for each mass track may be slightly above the true minimum. This introduces some fluctuations in the $\chi^{2}$ curves that would be smoothed out if the true minimum for each mass track were determined.

In order to more clearly represent the behavior of the weighted $\chi^{2}$, we have selected the minimum values of $\chi^{2}$ for each mass and plotted only those values (i.e., we trace the bottom edge of the $\chi^{2}$ values plotted in Figs. 3 and 4). In Figure 6 we show the minimum $\chi^{2}$ values (hereafter we refer to this ordinate as just $\chi^{2}$ ) for all of the models in our grid plotted against age, and in Figure 7 against mass. Lines connect $\chi^{2}$ values of similar composition and diffusion characteristics, as indicated in the legend. The model that best matches the BiSON $l=0,1$, and $2 p$-mode spectrum corresponds to the model with the lowest $\chi^{2}$.

The $Z=0.02, Y=0.27$ diffusion subgroup of the grid of models has the lowest values of $\chi^{2}$, although the subgroup of models at the same composition but calculated without diffusion have only slightly higher $\chi^{2}$ values. This is the result expected, since the best standard solar models have nearly this composition and do include the effects of $Y$ and $Z$ diffusion. The $Z=0.01$ and the 0.04 models all have higher $\chi^{2}$ values. The models with different helium abundances, the

TABLE 3

Solar Oscillation Data Range

\begin{tabular}{|c|c|c|c|c|c|c|}
\hline \multirow[b]{2}{*}{ OBSERVATIONS } & \multicolumn{2}{|c|}{$l=0$} & \multicolumn{2}{|c|}{$l=1$} & \multicolumn{2}{|c|}{$l=2$} \\
\hline & $\begin{array}{c}\nu \\
(\mu \mathrm{Hz})\end{array}$ & $n$ & $\begin{array}{c}\nu \\
(\mu \mathrm{Hz})\end{array}$ & $n$ & $\begin{array}{c}\nu \\
(\mu \mathrm{Hz})\end{array}$ & $n$ \\
\hline BiSON... & $1548-3984$ & $10-28$ & $1472-3914$ & $9-27$ & $1394-3977$ & $8-27$ \\
\hline VIRGO & $2093-4669$ & $14-26$ & $2157-3914$ & $14-27$ & $2218-3977$ & $14-27$ \\
\hline GONG 2001.1..... & $1957-4669$ & $13-33$ & $2157-4592$ & $14-32$ & $1945-4378$ & $12-30$ \\
\hline
\end{tabular}




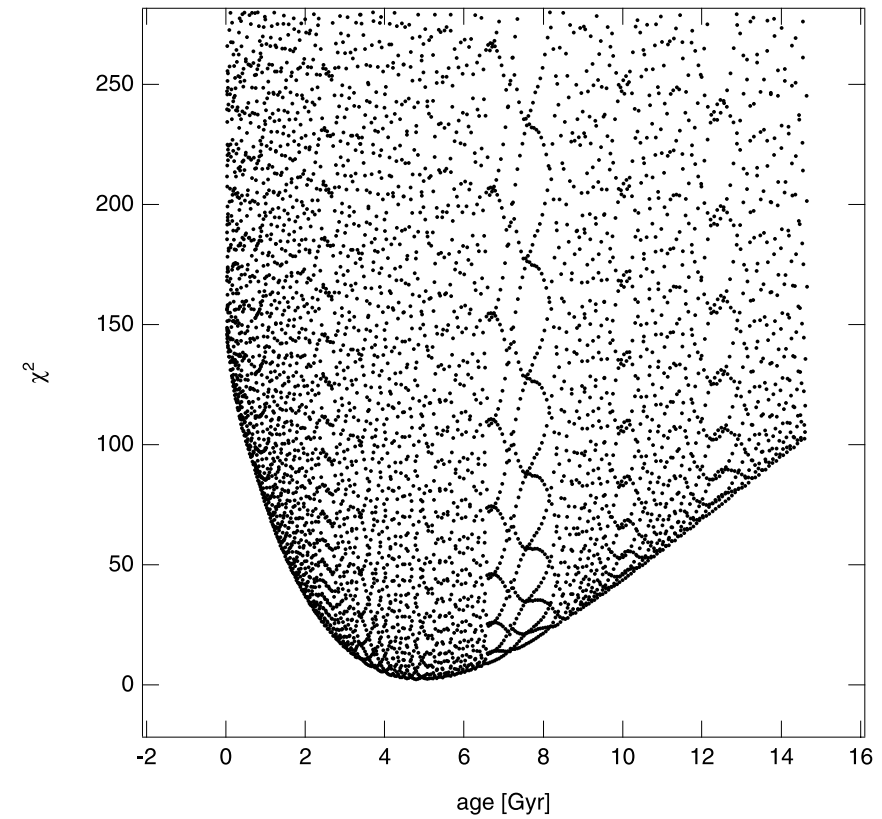

FIG. 3. $-\chi^{2}$ values calculated for BiSON data and the $Z=0.02, Y=0.27$ diffusion portion of the model grid, plotted as a function of age.

$Y=0.25,0.26$, and 0.27 at $Z=0.02$ (no diffusion) subgroup of models, do not show distinctly lower or higher values of $\chi^{2}$.

Without resorting to prior knowledge of the star being studied, one would conclude from the mass and age versus $\chi^{2}$ plots that the best model in the grid corresponds to $Z=0.02$, $Y=0.27$ with diffusion at a mass of $0.99 M_{\odot}$ and an age of 4.7 Gyr. The age is overestimated by $0.15 \mathrm{Gyr}(3 \%)$, and the

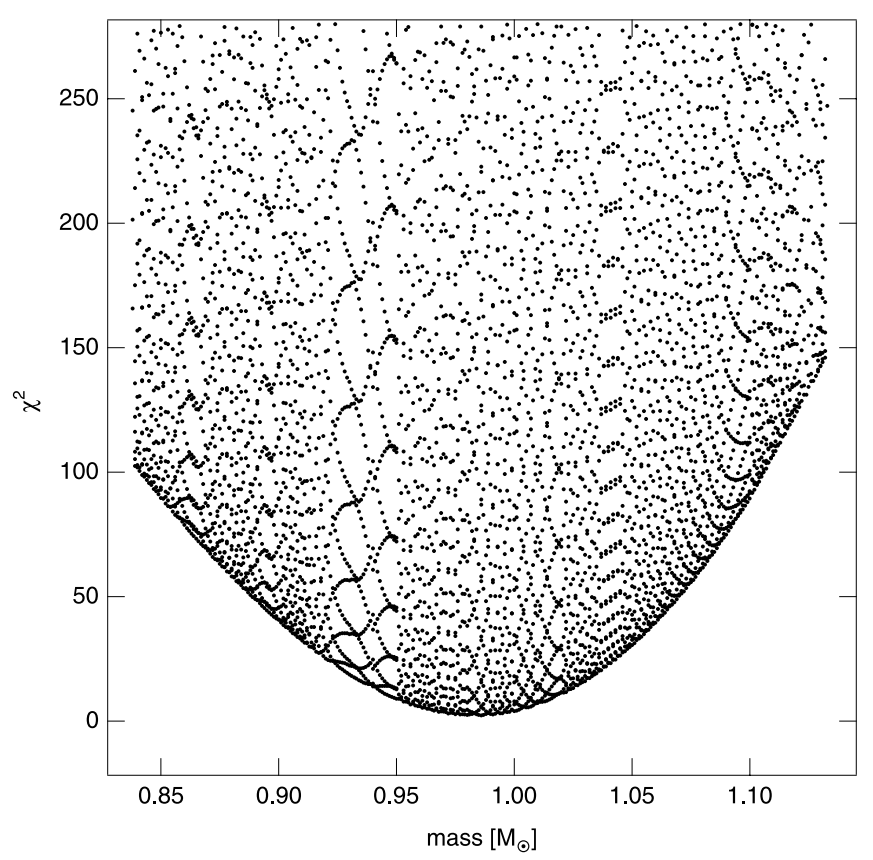

FIG. 4. $-\chi^{2}$ values calculated for BiSON data and the $Z=0.02, Y=0.27$ diffusion portion of the model grid, plotted as a function of mass. As in Fig. 3, only values of $\chi^{2}<1000$ are plotted. The $\chi^{2}$ values for masses less than $\sim 0.85 M_{\odot}$ and greater than $\sim 1.13 M_{\odot}$ are not computed because the spectra of the models do not match solar spectra well enough.

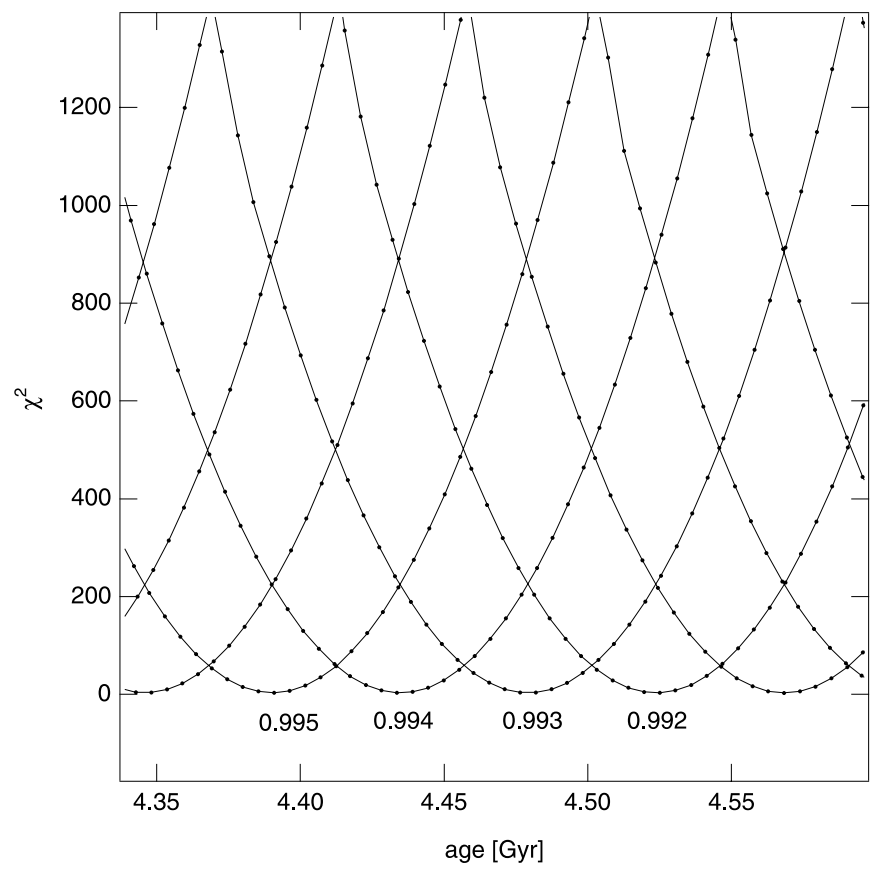

Fig. 5.-Zoomed-in portion of Fig. 3. Lines connect $\chi^{2}$ values of similar mass and changing age. Four of the curves are annotated with their corresponding model masses, in units of $M_{\odot}$.

mass underestimated by $0.01 M_{\odot}(1 \%)$. These discrepancies approximately quantify the error in the mode-matching method associated with the models themselves and their frequency calculation.

The systematic error in the model frequencies (frequencies of the standard solar model become higher than the Sun's frequencies as $n$ increases) leads to an overestimate of the age and a corresponding underestimate of the mass. For the standard solar model, the model is constrained by the solar mass, radius, and age, and this predicts higher values for the large spacing than observed. In our $\chi^{2}$ determination, the process is reversed; our model is constrained by the pulsation modes, and this predicts higher values for the age and lower values for the mass. We also note that our standard solar model includes an accurate model atmosphere, the Krishna Swamy empirical fit to the Sun's atmosphere. Our model grid is based on the less accurate, but more general, gray atmosphere in the Eddington approximation. This too adds uncertainty to the mass and age predictions when using only the models and oscillation frequencies as constraints.

The mode-matching method yields results that are sensitive to the effects of diffusion. The effect is minimal in mass; as we see in Figure 7 for $Z=0.02, Y=0.27$, the mass at which the nondiffusion $\chi^{2}$ curve is a minimum is very near the mass at which the (nearly overlapping) diffusion $\chi^{2}$ curve is a minimum. At $Z=0.04$, although the diffusion and nondiffusion $\chi^{2}$ curves do not overlap, their minima occur at nearly the same mass. The effect of diffusion is, however, visible in age, as shown in Figure 6. For the $Z=0.02, Y=$ 0.27 models, $Y$ and $Z$ diffusion lowers the age, as implied by the minimum in $\chi^{2}$, by approximately $1 \mathrm{Gyr}$. The effect is the same for the $Z=0.04$ models. The age of nondiffusion $Z=0.02, Y=0.27$ models are much more discrepant with the Sun's age than the diffusion models.

The effect of diffusion on age is understandable, given that when helium and heavy-element diffusion are included in the 


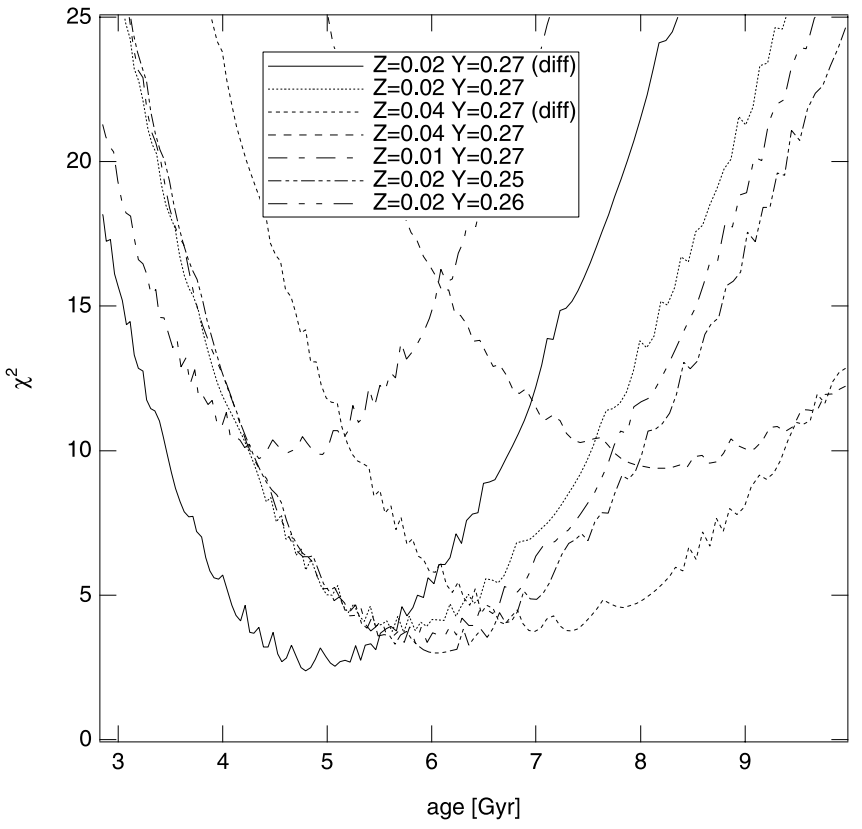

FIG. 6.-Bottom edge of the weighted $\chi^{2}$ values shown in Figs. 3 and 4, corresponding to the minimum value of $\chi^{2}$ for each model mass. These minimum $\chi^{2}$ values (hereafter referred to as $\chi^{2}$ values) are plotted as a function of age. Distinct line styles connect $\chi^{2}$ values from similar composition portions of the model grid. The oscillation data used are the $l=0,1$, and $2 p$-mode frequencies from BiSON.

standard solar model, the primordial abundance of helium and metals must be increased slightly above the observed surface abundance (as implied by $[Z / X]$ ). By the time the model has evolved to the age of the Sun, some of the metals and helium have drained out of the surface convection zone, thereby lowering their surface abundances compared to their abundances in the deep interior. The rate of nuclear burning is controlled by the mean molecular weight in the central regions of the Sun. Increasing the mean molecular weight in this

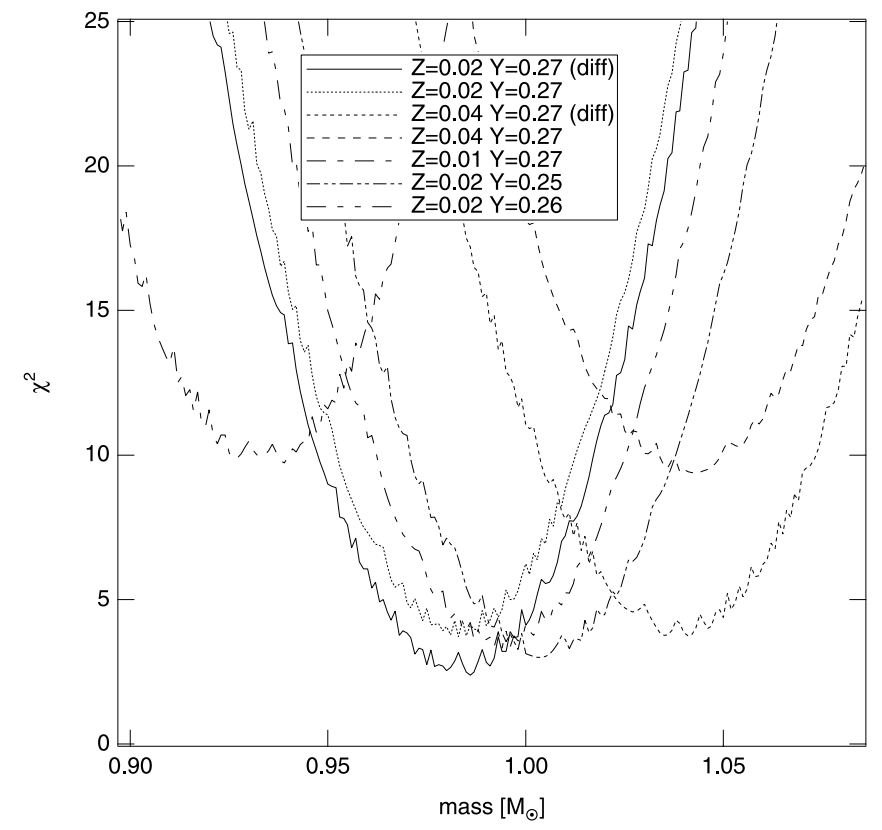

FIG. 7.- Similar to Fig. 6, except that $\chi^{2}$, i.e., minimum $\chi^{2}$, is plotted as a function of mass. region, which happens when $Y$ and $Z$ are increased, increases the rate of nuclear burning. The models with diffusion evolve more quickly and reach the Sun's observed position in the H-R diagram sooner, i.e., at a younger age, because they require higher abundances of $Y$ and $Z$ in the core (in order to match the observed surface abundances at the Sun's age) than nondiffusion models.

Figures 6 and 7 show that the abundance of helium has only a small effect on the mass location of the minimum in $\chi^{2}$ and a negligible effect on the age location of the minimum in $\chi^{2}$. Furthermore, the minimum value of $\chi^{2}$ itself appears unaffected by the abundance of helium in the models.

Unlike helium, the low- $l p$-mode frequencies, as revealed by $\chi^{2}$ curves, are sensitive to the metal abundance. Both the minimum values of $\chi^{2}$ and their mass and age location are affected. Of the $\chi^{2}$ curves for $Z=0.01,0.02$, and 0.04 , the lowest minimum in $\chi^{2}$ occurs for $Z=0.02$. We expect that adding more models to the grid at different $Z$ in this metallicity range would yield a well-defined minimum in $\chi^{2}$ as a function of $Z$.

In Figure 8 we show the $\chi^{2}$ versus mass curves for BiSON, GONG 2001.1, and VIRGO data, and in Figure 9 we show the $\chi^{2}$ versus age curves. Fitting quadratics to the GONG and VIRGO $\chi^{2}$ versus mass curves and then locating their minima gives masses equal to 0.973 and $0.978 M_{\odot}$, respectively. The widths of the $\chi^{2}$ curves, i.e., how sharply they dip to minimum, is a direct function of the uncertainty in their corresponding $p$-mode frequency determinations: the larger the uncertainty, the shallower the curves. The different locations of the $\chi^{2}$ minima provide a rough estimate of the uncertainties. The systematic shift to lower masses is caused by the errors in our models themselves (gray atmosphere and mixing-length theory), which have been slightly underestimated with $\sigma_{\text {mod. }}$. We can see from Table 3 that the BiSON data set extends to lower frequencies than the GONG and

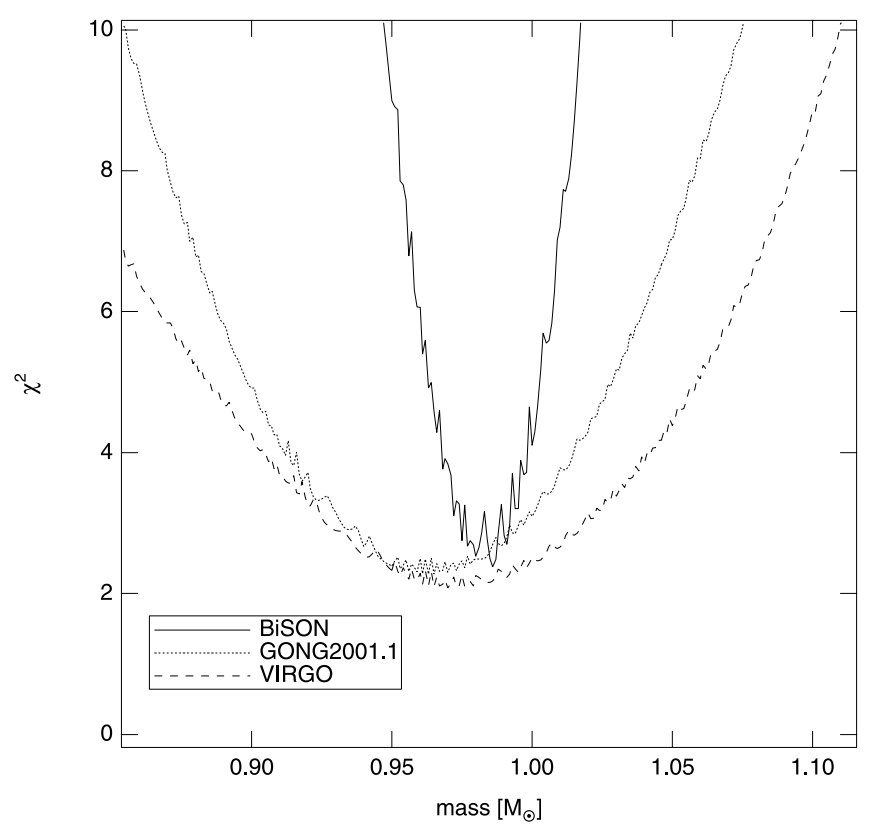

FIG. 8. $-\chi^{2}$ curves for the $Z=0.02, Y=0.27$ diffusion portion of the model grid, plotted as a function of mass for BiSON, GONG, and $\mathrm{SOHO/}$ VIRGO data. The BiSON curve is the narrowest because its data contain lower frequency modes, i.e., deeper penetrating modes, than the GONG and VIRGO data. The minima in the $\chi^{2}$ curves occur at masses slightly below $1 M_{\odot}$, suggesting that there exist systematic uncertainties in the models. 


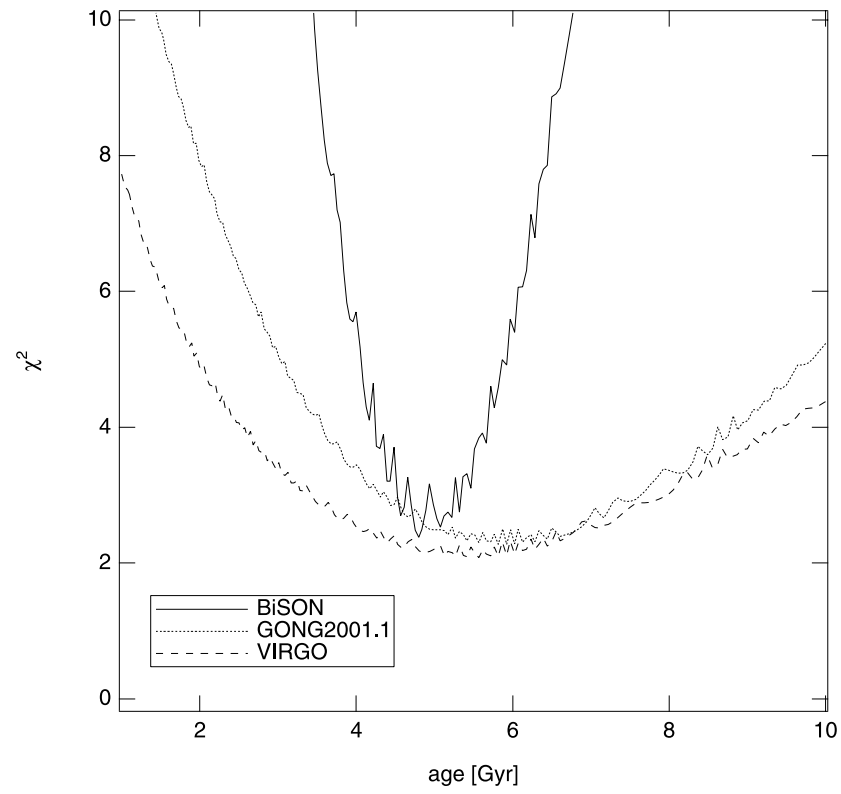

FIG. 9.- Similar to Fig. 8, except that $\chi^{2}$ curves are plotted as a function of age.

VIRGO data sets. Lower frequency $p$-modes are less affected by the outer layers than the higher frequency modes. The model uncertainties are much less for the lower frequency modes, with greater weight assigned to these lower frequencies. The $\chi^{2}$ values for BiSON data are, as a consequence, not contaminated as much by the more poorly modeled highfrequency modes as they are for the GONG and VIRGO data sets.

\subsection{Effects of Observational Uncertainty}

To study the effect of increasing observational uncertainty on the $\chi^{2}$ curves, we artificially add known values of Gaussian noise to the GONG 2001.1 frequencies. The $l=0,1$, and 2 GONG 2001.1 data have uncertainties of less than $0.2 \mu \mathrm{Hz}$ from 2000 to $3500 \mu \mathrm{Hz}$, with the error then increasing to $2 \mu \mathrm{Hz}$ by $4500 \mu \mathrm{Hz}$. We perturbed the GONG frequencies by adding random Gaussian-distributed perturbations of $\pm 1, \pm 2$, and $\pm 5 \mu \mathrm{Hz}$ (identified as Gauss \pm 1 , Gauss \pm 2 , and Gauss \pm 5 ). Regardless of the random noise added, in all but the original data set, we kept $\sigma_{\mathrm{obs}}=1 \mu \mathrm{Hz}$. In the case of the Sun, in which the model uncertainties are very low for the lowest frequency modes, increasing or decreasing $\sigma_{\text {obs }}$ shifts the $\chi^{2}$ curves up and down without affecting the shape of the curve. In general, however, the shape of the curve can be affected. Because we want to show how the $\chi^{2}$ curves respond to observational uncertainties, regardless of the values of $\sigma_{\text {obs }}$ quoted, we have kept the observational error parameter fixed.

In Figures 10 and 11 we show the mass and age $\chi^{2}$ curves for these data sets, along with the original GONG 2001.1 data set. As the noise in the data is increased, the width of the $\chi^{2}$ curves also increases. For frequencies accurate to $\pm 1 \mu \mathrm{Hz}$, the best mass and age are well defined, but for the Gauss \pm 2 and the Gauss \pm 5 data sets, the minimum in $\chi^{2}$ is more poorly defined. All curves span only the range $0.84-1.12 M_{\odot}$, since the spectra of all models beyond this mass range do not match the observational data set (for which, as previously defined, a

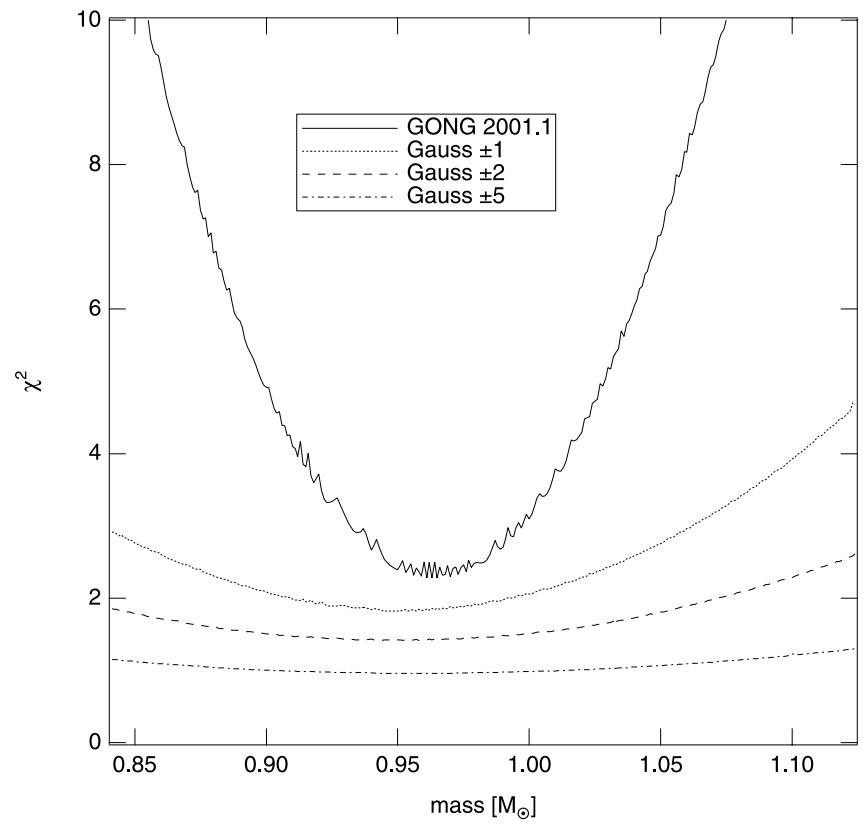

FIG. 10.- To study the effects of noise in the frequency data, Gaussiandistributed random noise was added to the GONG frequency data. Three data sets were generated: Gauss \pm 1 with $\pm 1 \mu \mathrm{Hz}$ of random Gaussian-distributed noise added, Gauss \pm 2 with $\pm 2 \mu \mathrm{Hz}$ of random Gaussian-distributed noise added, and Gauss \pm 5 with $\pm 5 \mu \mathrm{Hz}$ of random Gaussian-distributed noise added. The $\chi^{2}$ curves for the $Z=0.02, Y=0.27$ diffusion portion of the model grid are plotted as a function of mass for these three data sets along with the original GONG data set, whose frequencies have uncertainties $\sigma_{\text {obs }}$ of less than $\pm 0.1 \mu \mathrm{Hz}$

match requires all but five frequencies in the observed spectra to lie within $\Delta \nu / 4$ of the model frequencies).

Although poorer quality data can constrain the models, they do so at a level below that of conventional observational constraints. To help further constrain the models, the

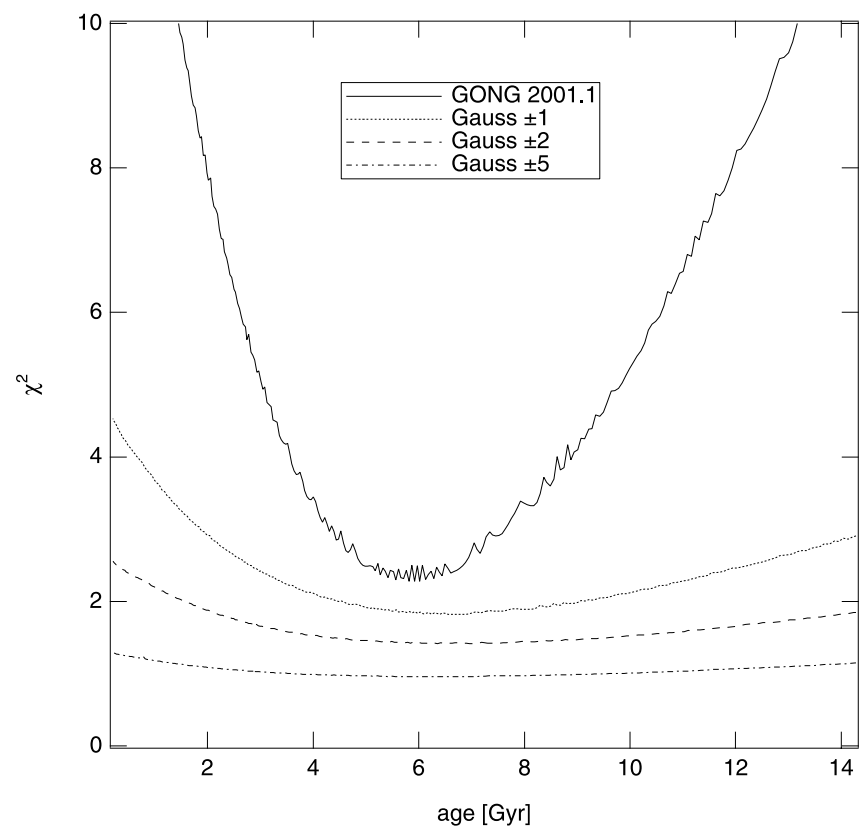

FIg. 11.-Similar to Fig. 10, except that $\chi^{2}$ curves are plotted as a function of age 
oscillation mode frequencies need to be observed with an accuracy of at least $\pm 1 \mu \mathrm{Hz}$.

\subsection{Using $l=0$ and 1 Data Only}

In all the tests carried out so far, we have used the $l=0,1$, and 2 modes. One might guess that restricting the data set to just $l=0$ (radial only), or $l=0$ and 1 modes will severely limit the utility of the data to resolve mass and age. This is not the case. To show this, we took the Gauss \pm 1 data set and removed the $l=2$ modes, then also the $l=1$ modes, from the data set and applied the mode-matching routines to these two data sets. Although the curves are shallower, both the radial only and the $l=0$ and 1 data sets have clearly defined minima, as shown in Figures 12 and 13. This result is important, since stars on the subgiant branch are expected to have only observable radial modes (Guenther et al. 2000).

\subsection{High Correlation between Parameters}

When the $\chi^{2}$ values are plotted as a function of mass and age together, the lowest values of $\chi^{2}$ (e.g., < 10) lie within a very narrow line in mass and age (so narrow that we are unable to produce a visually useful contour plot). Similarly, the lowest values of $\chi^{2}$ define a very narrow line in effective temperature and luminosity. That is to say, when the models in the grid are constrained optimally by the oscillation spectra, a unique relation between mass and age, or effective temperature and luminosity, is defined. At low $\chi^{2}$ the mass and age are very highly correlated, and so are the effective temperature and luminosity. In Figure 14 we show the correlation between mass and age for low values of $\chi^{2}$ (taken at the minimum value in $\chi^{2}$ at each age) for BiSON data. In Figure 15 we show the correlation between effective temperature and luminosity.

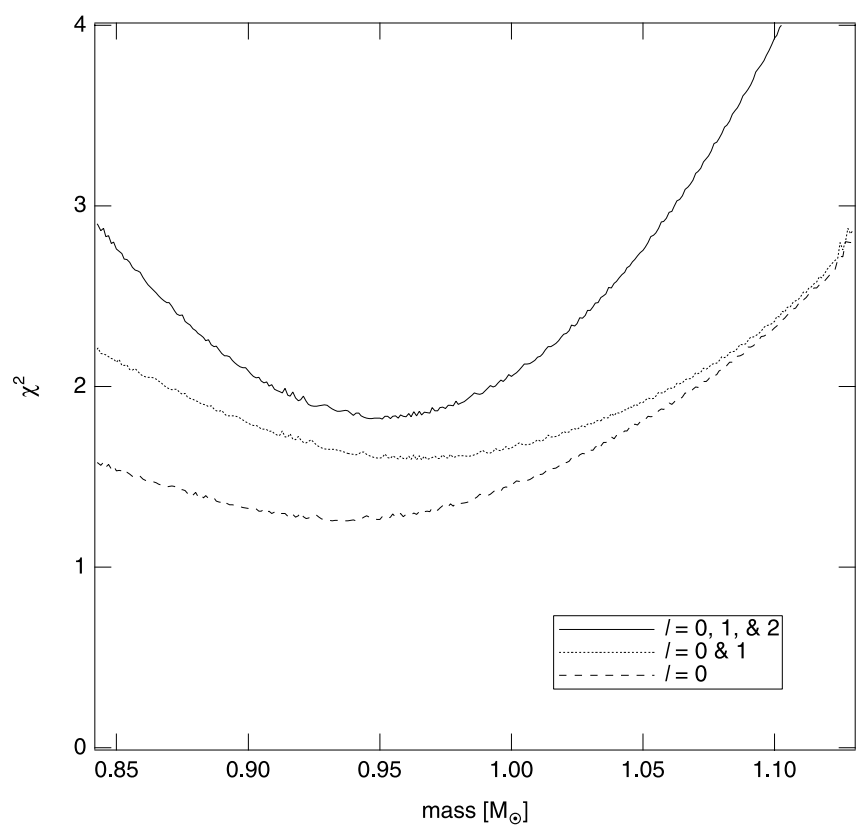

FIG. 12.-Because stars are observed as points of light, asteroseismic observations will only resolve the lowest $l$-valued $p$-modes. To study the effect of only using $l=0$ modes and $l=0,1$ modes, we take the Gauss \pm 1 data set and generate two restricted sets, one containing only the $l=0$ modes and the other containing only the $l=0,1$ modes. The $\chi^{2}$ curves for the $Z=0.02$, $Y=0.27$ diffusion portion of the model grid are plotted as a function of mass for these two data sets along with the original Gauss \pm 1 data set.

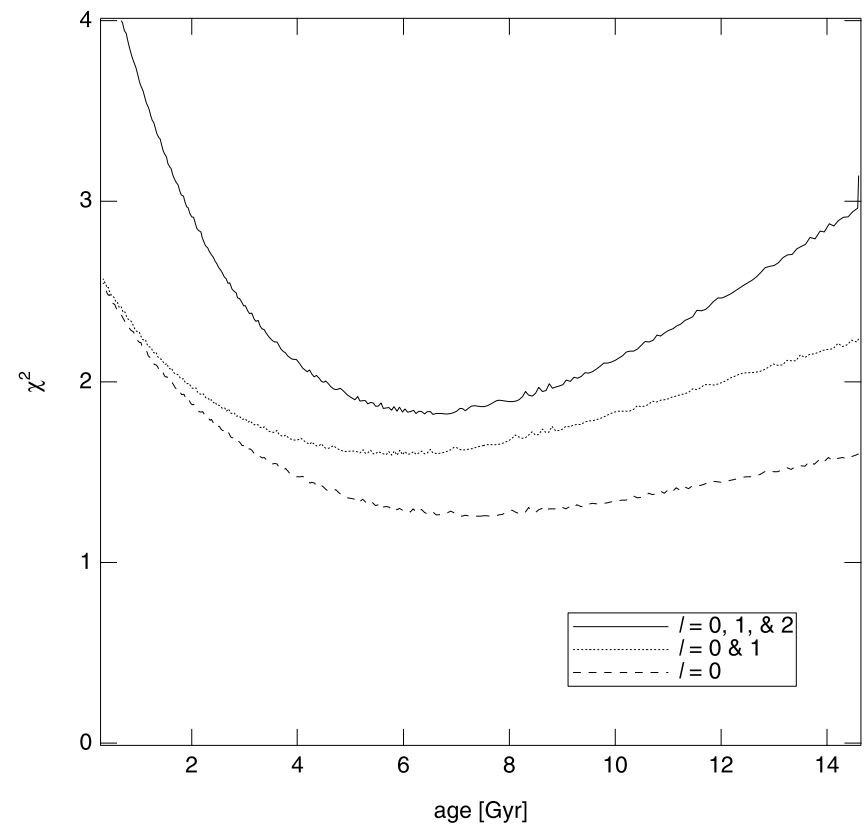

FIG. 13.-Similar to Fig. 12, except that $\chi^{2}$ curves are plotted as a function of age.

The two curves in each figure correspond to $Z=0.02, Y=$ 0.27 (diff) and $Z=0.04, Y=0.27$ (diff).

\section{4. $\alpha$ CEN A}

To provide a more interesting test, we apply the modematching method to recent ground-based observations of $p$-mode oscillations on $\alpha$ Cen A. Bouchy \& Carrier (2002) made Doppler shift observations of $\alpha$ Cen A over 13 nights and identified 28 individual $l=0,1$, and $2 p$-modes. They compared the large and small spacings with existing models by Morel et al. (2000) and Guenther \& Demarque (2000) and

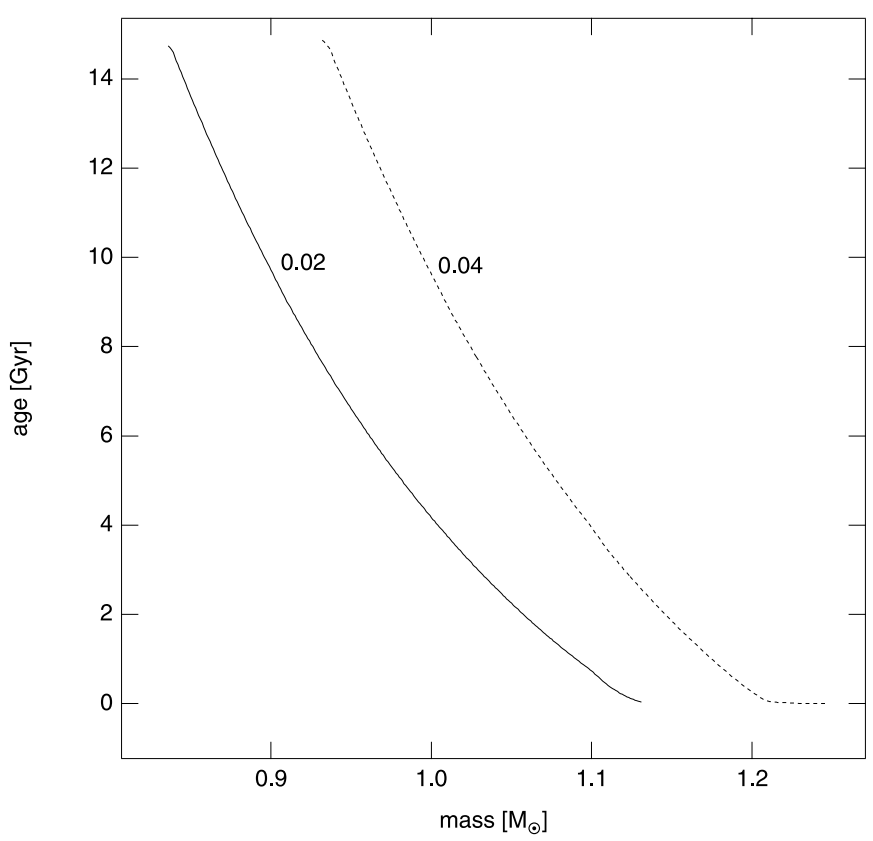

FIG. 14.-Correlation between the mass and the age of the models that have low $\chi^{2}$ values. BiSON data are used. 


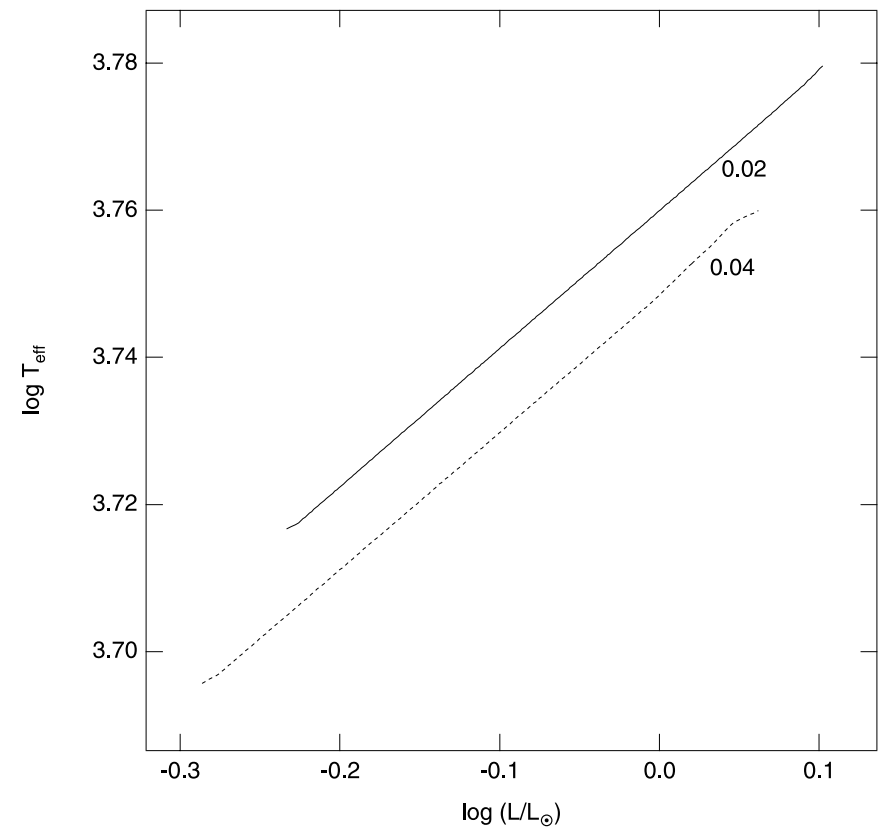

FIG. 15.-Correlation between the luminosity and the effective temperature of the models that have low $\chi^{2}$ values. BiSON data are used.

found the observations to be in general agreement with these models, falling somewhat in the middle of the two with regard to mass and age. Thévenin et al. (2002) carried out a traditional modeling analysis of $\alpha$ Cen $\mathrm{A}$ and $\mathrm{B}$, similar to that of Guenther \& Demarque (2000) but utilizing slightly different nonoscillation constraints and using the Canuto \& Mazzitelli (1991, 1992) prescription for the mixing-length theory. They compared the oscillation spectra of their models with the observed large and small spacings of Bouchy \& Carrier (2002). They were unable to completely reconcile the two.

The oscillation data as published in Bouchy \& Carrier (2002) without their l-identifications were processed by the mode-matching program using the same grid as described in previous sections of this paper. We begin by considering the three-dimensional plot of $\chi^{2}$ versus mass and age shown in Figures $2 a$ and $2 b$ for the $Z=0.04, Y=0.27$ diffusion portion of the grid. As discussed in $\S 2.7$, this plot shows that the mass and age of the best-fitting models (models with $\chi^{2}<5$ ) lie on a curved surface that defines a direct correlation between mass and age. We further note that there is a welldefined minimum $\chi^{2}$. Curiously, a second surface appears for $\chi^{2}>8$. Examination of the models themselves reveals that the smaller surface corresponds to models that have a convective core or had a convective core during their evolution, and the larger surface corresponds to models that never develop convective cores during their evolution. As discussed by Guenther \& Demarque (2000), models of $\alpha$ Cen A are precariously balanced between developing a convective core or not during their evolution depending on initial conditions. Models that do have a convective core at some time during their evolution take longer to evolve to a given position in the $\mathrm{H}-\mathrm{R}$ diagram than models that do not. Because the existence or nonexistence of the convective core is very sensitive to composition and mass, the age of the model can change abruptly as a function of mass and composition in this region of the H-R diagram. The best-fitting models for $\alpha$ Cen A correspond to models that do not have a convective core. That is, the oscillation data unambiguously rule out models that develop convective cores during their evolution.

Figures 16 (entire grid) and $17(Z=0.01,0.02$ [diff], and 0.04 [diff]) show the $\chi^{2}$ curves for mass and age, respectively. The $Z=0.04 \chi^{2}$ curves have well-defined minima. The $Z=$ $0.02 \chi^{2}$ curves show spikes and step-function jumps. The $Z=$ $0.01 \chi^{2}$ curves are not as nicely defined as for the Sun. For the $Z=0.04$ curves, which correspond most closely to the observed metallicity of $\alpha$ Cen A $(Z / X=0.041 \pm 0.002)$, the $\chi^{2}$ minima are near $1.15 M_{\odot}$ and 6 Gyr. For comparison, with $T_{\text {eff }}=5770 \pm 50 \mathrm{~K}, \log \left(L / L_{\odot}\right)=0.1969 \pm 0.017, Z / X=$ $0.041 \pm 0.002$, and $M=1.1015 \pm 0.008 M_{\odot}$, Guenther \& Demarque (2000) derive an age for the system of $7 \pm 1$ Gyr. The $\chi^{2}$ curves do appear to be consistent with conventional observations and models. The $Z=0.02$ curves show minima at lower masses and higher ages. Needless to say, the grid resolution in metallicity needs to be greater in order to infer the mass and age of $\alpha$ Cen A from $\chi^{2}$ curves that are near the observed metallicity $(Z \simeq 0.034)$ of $\alpha$ Cen A.

The spikes and step-function jumps in the $Z=0.02 \chi^{2}$ curves occur because the models that had convective cores have different oscillation frequencies from models that do not, even though the models themselves may be very close to each other in the model grid. They do not appear in the $Z=0.04 \chi^{2}$ curves simply because the convective core models do not overlap the nonconvective core models at low values of $\chi^{2}$.

We note that the diffusion and nondiffusion models do not show significantly distinct $\chi^{2}$ curves; hence, the current oscillation data by themselves cannot be used to support the case for the existence of $Y$ and $Z$ diffusion. With other observational constraints and a denser grid along the $Z$-dimension, it may be possible to distinguish the nondiffusion case from the diffusion case.

In Figure 18 we plot in an H-R diagram the location of the $\chi^{2}$ minima along each mass track. Recall (see $\S 3.4$ ) that near

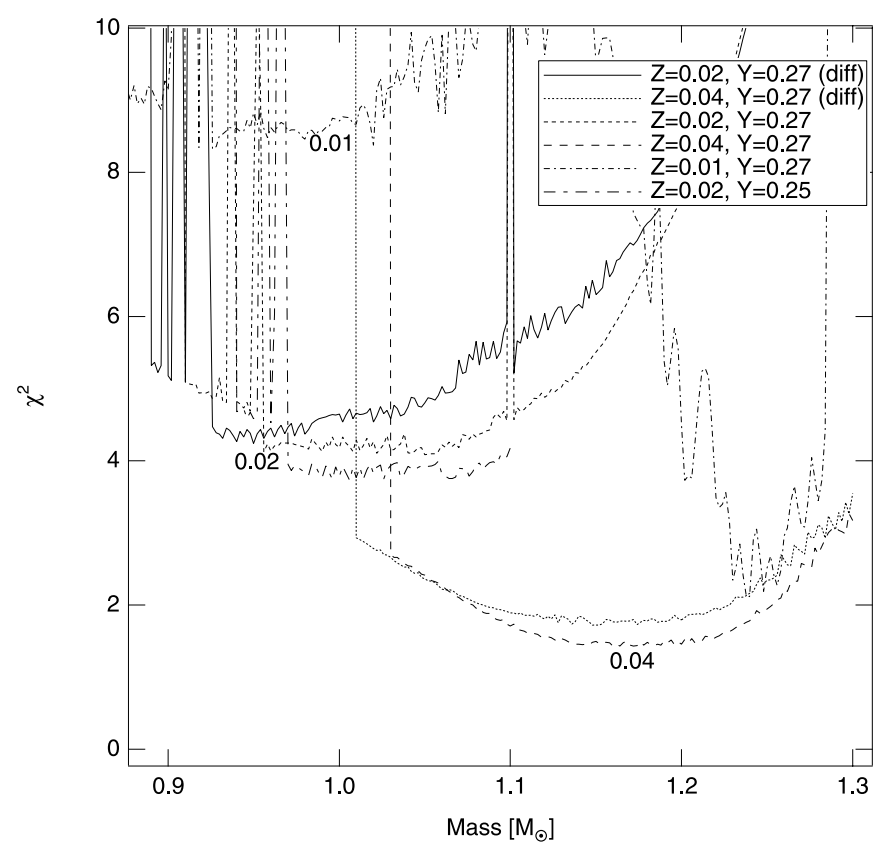

FIG. 16. - $\chi^{2}$ curves plotted as a function of mass for the Bouchy \& Carrier (2002) $\alpha$ Cen A oscillation data (see text). The $Z=0.04$ portion of the model grid produces the best-fitting oscillation spectra (as indicated by the low values of $\chi^{2}$ ) for masses around $1.15 M_{\odot}$. 


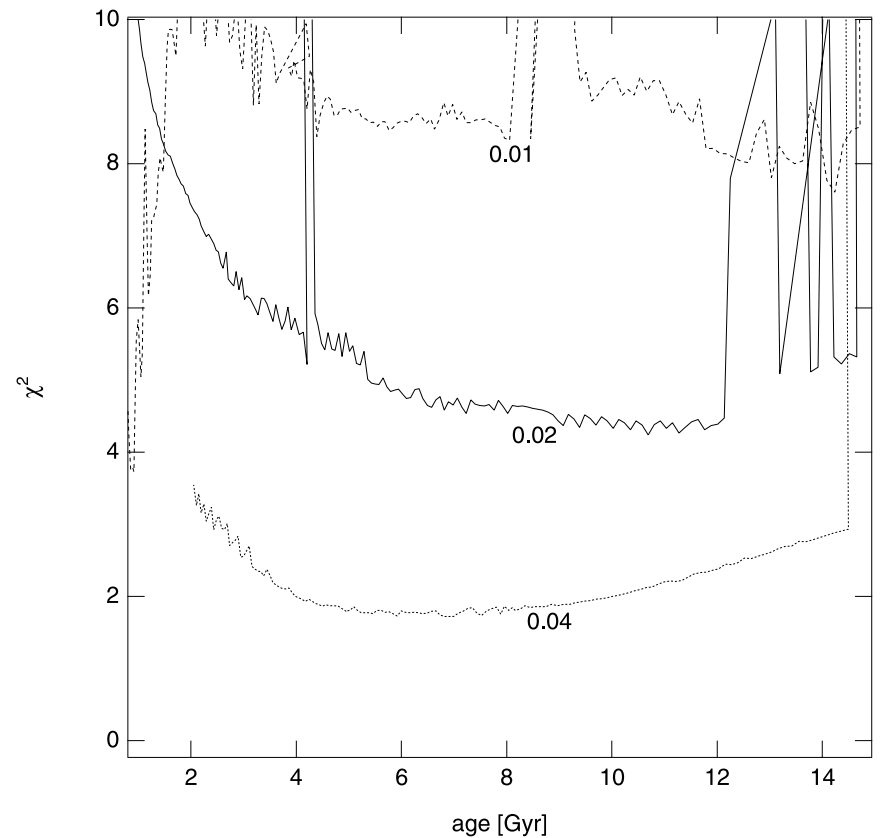

Fig. 17.- Similar to Fig. 16, except that $\chi^{2}$ curves are plotted as a function of age for the $(Z=0.02, Y=0.27),(Z=0.02, Y=0.27$, diffusion), and $(Z=0.04, Y=0.27$, diffusion) compositions.

the $\chi^{2}$ minima, the luminosity is highly correlated with the effective temperature. For each composition, a single curve is defined in the H-R diagram. The location of $\alpha$ Cen $\mathrm{A}$ as implied by conventional observations is also indicated in the diagram. The $Z=0.04$ curve passes through $\alpha$ Cen A's position, while the $Z=0.02$ curve does not. As noted above, Guenther \& Demarque (2000) find that when diffusion of $Y$ and $Z$ are included in the models, the ZAMS metallicity of $\alpha$ Cen $\mathrm{A}$ is $\sim 0.034$, which is consistent with the results of the $\chi^{2}$ curves.

In Figure 19 we show an echelle diagram of the observational data along with one of the lower $\chi^{2}$ model matches $\quad\left(\right.$ mass $=1.148 M_{\odot}$, age $=6.99316 \mathrm{Gyr}, \chi^{2}=$ 1.7202). The model oscillation frequencies fit the observed frequencies to better than $1 \%-2 \%$. The fit is best at lower frequencies, with a systematic shift to greater differences at higher frequencies. The higher frequencies are more poorly fitted because the model uncertainties are larger at higher frequencies. The shift is most likely caused by uncertainties in the outer layers of the model. The oscillation data for $\alpha$ Cen A, in particular the frequency shift, similar to that seen for the Sun, will provide much needed information about the general behavior of convection in stars.

The oscillation spectrum of the model used in Figure 19 is a significantly better match to the observed spectrum of $\alpha$ Cen A than any of the oscillation spectra of models found from conventional methods (e.g., Guenther \& Demarque 2000; Thévenin et al. 2002). Here we are showing how the oscillations by themselves can be used to infer the mass and age of $\alpha$ Cen A. Needless to say, a comprehensive analysis of $\alpha$ Cen A would demand folding together all observational constraints.

\section{5. $\alpha$ UMa A}

We have, so far, only considered solar-type stars. Tentative $p$-mode oscillations have been identified on $\alpha$ UMa A by Buzasi et al. (2000) using the star camera on the failed Wide

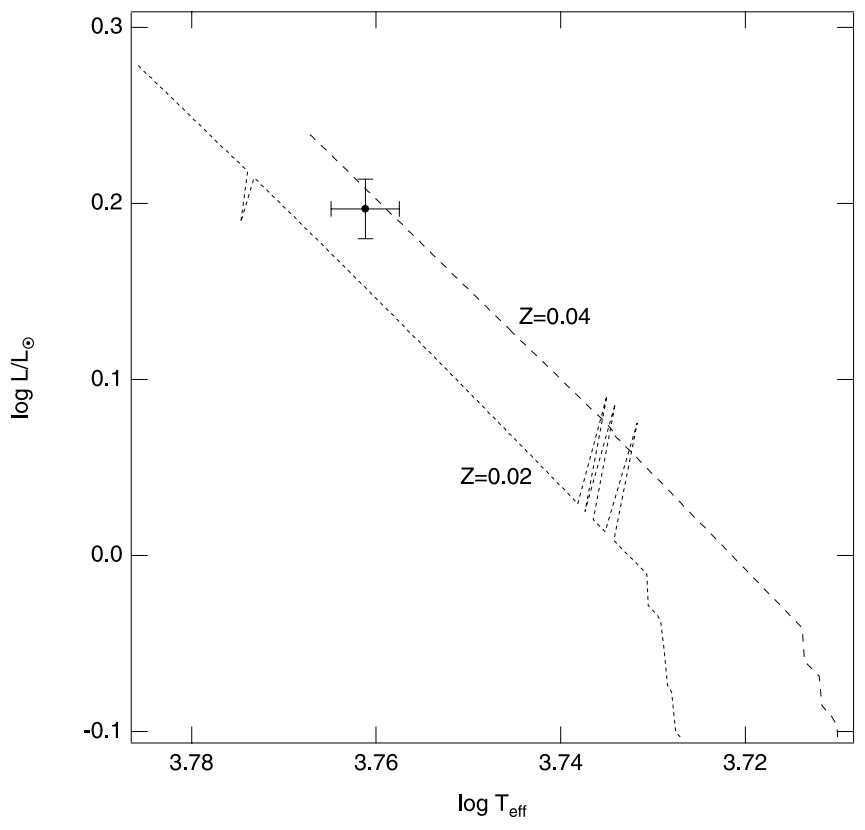

FIG. 18.-Along each stellar model mass track $\chi^{2}$ reaches a minimum. The loci of all the H-R diagram positions of the models for which the $\chi^{2}$ reach a minimum for the $\alpha$ Cen A oscillation data are shown here for the $Z=0.02$, $Y=0.27$ diffusion portion of the model grid and the $Z=0.04, Y=0.27$ diffusion portion of the model grid. The position of $\alpha$ Cen A in the H-R diagram is also plotted.

Field Infrared Explorer satellite. $\alpha \mathrm{UMa} \mathrm{A}$ is the primary star, of spectral type K0 III, in a visual binary system. The secondary is classified as an F7 V star with a mass estimated between 1 and $2 M_{\odot}$. The total mass of the binary system is approximately $6 M_{\odot}$ (Söderhjelm 1999); hence, the mass $\alpha$ UMa A is estimated to be between 4 and $5 M_{\odot}$. Guenther et al. (2000) constructed several models constrained by nonoscillation observations and compared the oscillation

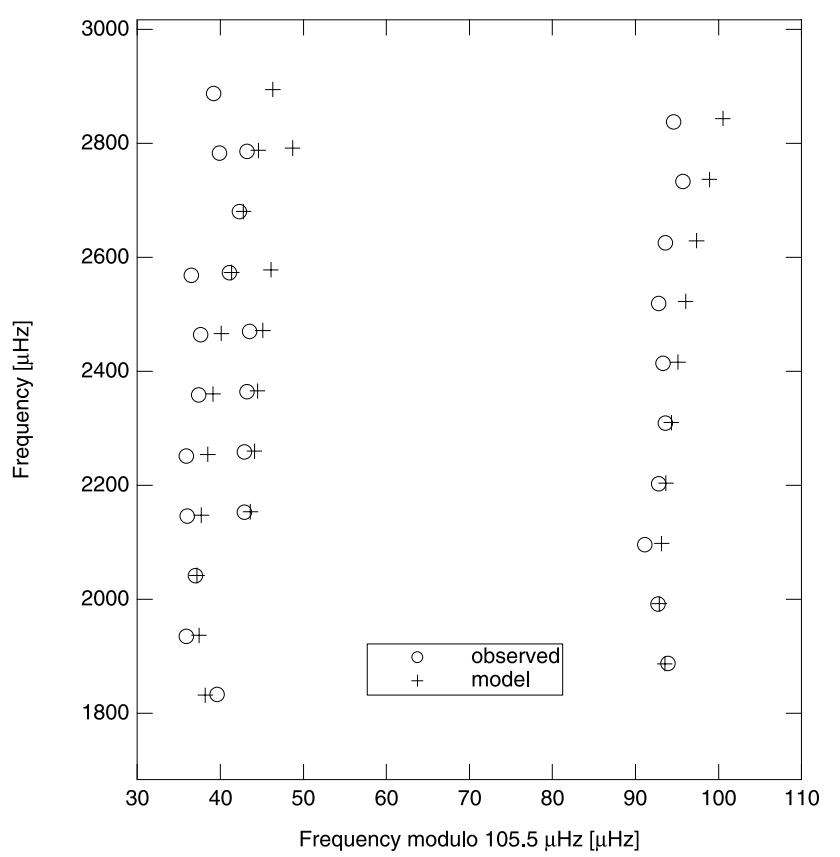

FIG. 19.-Echelle diagram of the observed $p$-mode frequencies for $\alpha$ Cen A (Bouchy \& Carrier 2002) and the frequencies of a matching model with low $\chi^{2}\left(\operatorname{mass}=1.14800 M_{\odot}\right.$, age $\left.=6.99316 \mathrm{Gyr}, \chi^{2}=1.7202\right)$. 
spectra of the models with the observed spectrum. The spectra of their models were in general agreement with the observations, but no definitive match could be made between the modes, and only weak conclusions could be drawn to support the validity of the observations.

We have run the observed frequencies for $\alpha$ UMa A through our mode-matching software to see if a more precisely matched model spectrum could be identified. We only considered the $Z=0.02, Y=0.27$ no diffusion portion of the grid because it is the only part of the grid that extends to the predicted mass of $\alpha$ UMa A. We also only looked at the radial modes, since the nonradial mode frequencies are not regularly spaced (see the discussion in Guenther et al. 2000). Following a trial run, we decided to decrease the mode-matching parameter NREJECT to 1 , since there are only 10 modes identified by Buzasi et al. (2000) to begin with.

With this mode-matching criteria, models between 3.9 and 4.6 $M_{\odot}$ possess spectra that match 9 of the 10 oscillation frequencies. The $\chi^{2}$ versus mass and age plots for $\alpha$ UMa A are shown in Figures 20 and 21. The mass and age of $\alpha$ UMa A implied by the oscillation data, and assuming $Z=0.02$, $Y=0.27$ (no diffusion), are $4.55 M_{\odot}$ and 0.11 Gyr. Table 4 lists the observed frequencies and the model frequencies $(l=0)$ for the best-matched model $\left(M=4.552 M_{\odot}\right.$, age $=$ $0.1104 \mathrm{Gyr})$. The observed lowest frequency mode was not matched in any of the minimum $\chi^{2}$ models. The abrupt change in $\chi^{2}$ at the end of the mass range (and beginning of the age range) is a consequence of the increasing rate of evolution (relative differences between models increases) as the mass increases. Based on Taylor's (1999) spectroscopic determination of $[\mathrm{Fe} / \mathrm{H}]$ and an assumed Galactic helium enrichment ratio of $\Delta Y / \Delta Z=2.5$, Guenther et al. (2000) calculate that the metallicity of $\alpha$ UMa $\mathrm{A}$ is $Z=0.0124$ and that the helium abundance is $Y=0.261$. We believe a lower mass would be determined if the $Z$ of our grid used here were closer to the derived $Z$ for $\alpha$ UMa A.

The well-defined minima in the $\chi^{2}$ curves, the closeness of the best-fit model spectra to the observed spectrum (less the

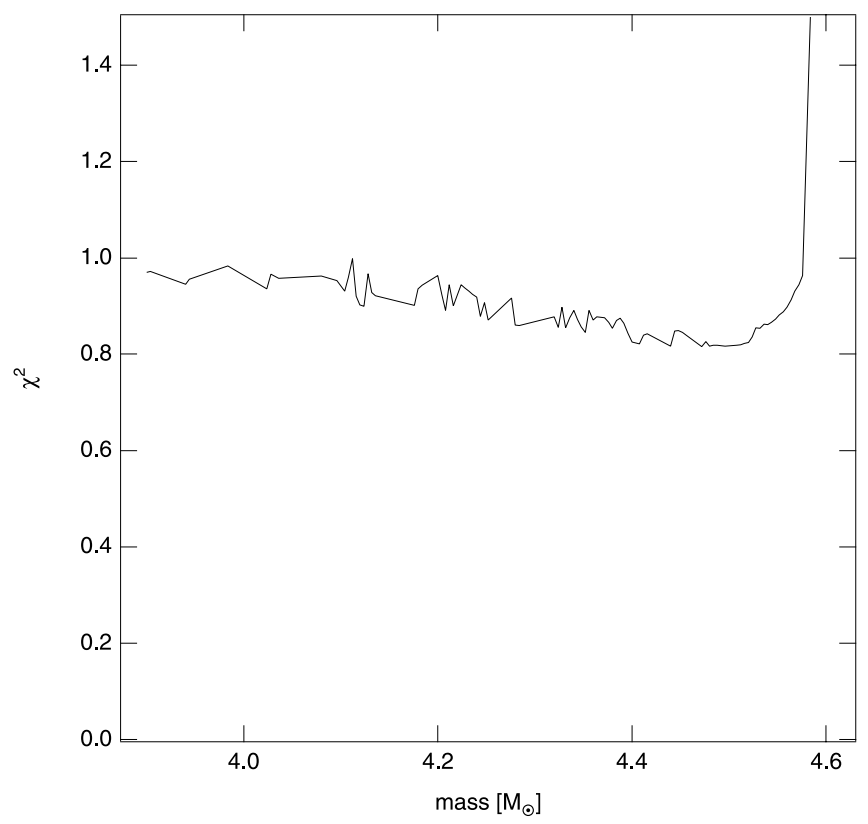

FIG. 20. $-\chi^{2}$ curve as a function of mass for the $\alpha$ UMa oscillation data of Buzasi et al. (2000). The curve is based on the $Z=0.02, Y=0.27$ (no diffusion) portion of the grid.

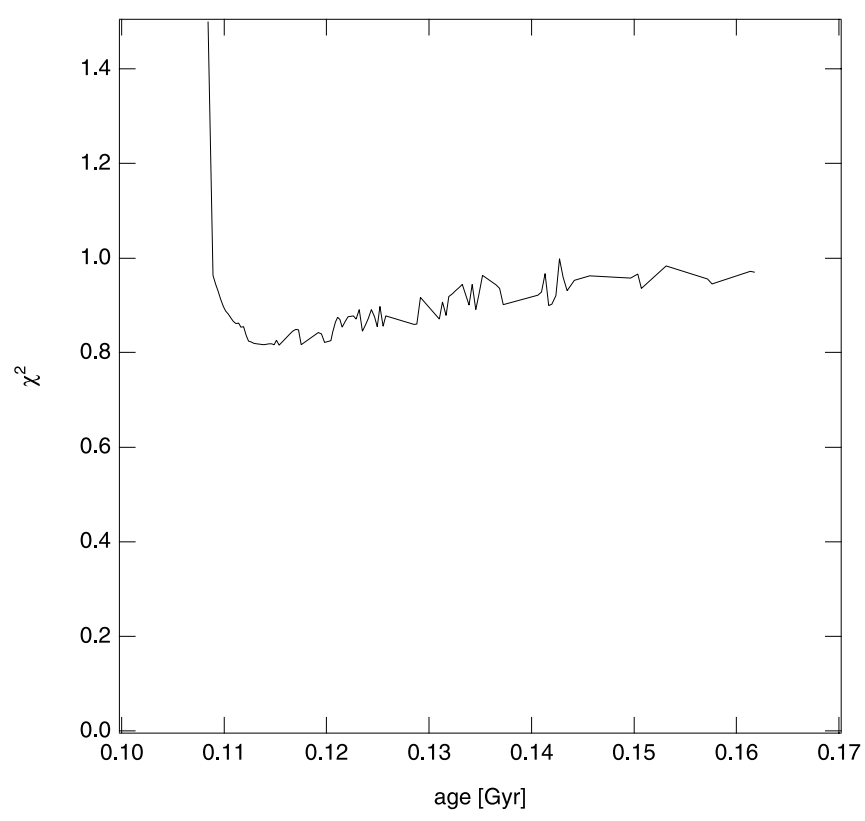

FIG. 21. $-\chi^{2}$ curve as a function of age for the $\alpha$ UMa oscillation data of Buzasi et al. (2000). The curve is based on the $Z=0.02, Y=0.27$ (no diffusion) portion of the grid.

lowest frequency mode), and the reasonableness of the derived mass and age provide theoretical support for the $p$-mode observations by Buzasi et al. (2000) of $\alpha$ UMa A. Furthermore, the success of the method confirms the robustness of the modematching method at higher masses.

\section{SUMMARY AND CONCLUSIONS}

We have introduced a method to study stellar oscillation data that utilizes an extensive grid of models parameterized by mass, age, and composition. The method quantifies how well an observed $p$-mode spectrum matches the spectra of the models using a $\chi^{2}$ measure. The $\chi^{2}$ values permit a range of bestmatching models, and the uncertainties in their parameters, to be identified. The best-matched models, as determined by the mode-matching method, are constrained exclusively by the oscillation data. As a consequence, the predictive abilities of the oscillation data are isolated from other observational constraints such as mass, radius, and luminosity.

The grid of models is necessarily very dense, and hence a significant number of stellar models need to be computed in order to cover a large mass, age, and composition range. Here we have presented initial test results on a limited grid centered around solar-type stars.

TABLE 4 $\alpha$ UMA A Frequencies

\begin{tabular}{ccr}
\hline \hline$\nu_{\text {obs }}$ & $\nu_{\text {model }}$ & $n$ \\
\hline $2.030 \ldots \ldots$. & $\ldots$ & $\ldots$ \\
$5.170 \ldots \ldots$. & 5.114 & 0 \\
$7.950 \ldots \ldots$. & 8.464 & 1 \\
$11.410 \ldots \ldots$ & 11.400 & 2 \\
$14.970 \ldots \ldots$ & 14.276 & 3 \\
$18.150 \ldots \ldots$ & 17.442 & 4 \\
$20.740 \ldots \ldots$ & 20.328 & 5 \\
$22.390 \ldots \ldots$ & 23.064 & 6 \\
$34.920 \ldots \ldots$ & 35.050 & 10 \\
\hline
\end{tabular}


We have tested the mode-matching method on solar data for which we know the observational data to be of high quality, and we know the mass and age of the Sun well enough to evaluate how well the mode-matching method works. The $\chi^{2}$ curves have well-defined minima. The accuracy of the values of mass and age predicted by the mode-matching method appears to be limited by the accuracy of our models themselves. The well-known weakness in the mixing-length theory used to describe the outermost layers of the convective envelope (the superadiabatic layer), the fact that our oscillation code cannot calculate nonadiabatic effects associated with turbulence, and the use of a gray atmosphere means that there is a systematic error in all our models and their spectra that increases with frequency. The most accurate results are obtained with BiSON data that extend to $1500 \mu \mathrm{Hz}$. GONG and VIRGO data do not extend as low in frequency, and hence are more affected by this inherent weakness in our grid of model spectra. Regardless, we find that the oscillation data alone with uncertainties of the order of $\pm 1 \mu \mathrm{Hz}$ can be used to constrain the age and mass to a few percent, even when the data are restricted to radial-only modes.

Although our grid has only a few distinct compositions, the results suggest that the metallicity of a star can also be determined by looking for the minimum in $\chi^{2}$ along this dimension of the grid. Our initial tests of trying to isolate the abundance of helium are not as promising. The abundance of helium does affect the location of the $\chi^{2}$ minima in mass and age, but the absolute value of the $\chi^{2}$ is unaffected; hence, we cannot pick out the minimum in $\chi^{2}$ along this dimension of the grid.

The sensitivity of the mode-matching method is especially revealed in its ability to distinguish between models with and without $Y$ and $Z$ diffusion. Models with diffusion have lower $\chi^{2}$ values, and their $\chi^{2}$ predictions of mass and age are closer to the Sun's true values.

If the systematic uncertainties in the model frequencies caused by the poor modeling of both convection and the atmospheres could be accounted for, then lower model uncertainties could be used that would yield narrower $\chi^{2}$ curves. On the other hand, the existence of this discrepancy makes it clear that stellar seismology is going to be extremely useful in fine-tuning the physics of the outer layers of our stellar models.

When applied to the observed oscillation data for $\alpha$ Cen A (Bouchy \& Carrier 2002), the mode-matching method produces an unambiguous range of matched models from the grid. The stellar models of $\alpha$ Cen A constrained entirely by the oscillation data are within the uncertainties of stellar models of $\alpha$ Cen A constrained by conventional observations, and the results indicate that the metal abundance can also be constrained when the grid resolution in $Z$ is increased. A more detailed analysis of the system will require folding in conventional observational constraints and their uncertainties, a task that is made easier by the ability of the mode-matching method to isolate the effect of the uncertainties in the oscillation data and the models.

The mode-matching method when applied to the oscillation data for the subgiant $\alpha$ UMa (Buzasi et al. 2000) was able to determine unambiguously the star's mass and age. Previous analysis of the oscillation data by Guenther et al. (2000) using conventional modeling methods were not as successful in validating the observations.

The mode-matching method cannot perform better than the models themselves. As shown in the tests using solar oscillation data, and noted in our discussion on the calibration of the model frequency uncertainties, the model uncertainties increase from less than $\pm 0.01 \%$ to $\pm 0.3 \%$ as $n$ ranges from 10 to 30 . We know from oscillation studies of our solar models that the superadiabatic layers are not accurately modeled using mixing-length theory. We also know that a good atmosphere model is required to obtain accurate $p$-mode frequencies at higher $n$. Since all $p$-mode frequencies are affected by the outer layers of the models, with the effect increasing with $n$, it may be possible to correct for the systematic shift in frequencies, thereby decreasing the effective uncertainties in the mode-matching method. We believe that up to an order of magnitude reduction in the uncertainties may be possible.

In the immediate future, along with extending the grid, we propose to modify the mode-matching software to search for the best matches of weighted combinations of $p$-mode frequencies. The weighted combinations would be similar to the weightings used to generate inversion kernels that pick out specific depths in a star. In this manner, we would use the mode-matching algorithms not only to find the best-fitting models but also to identify the regions in the interior of the model where the fit is poor.

Because the mode-matching routines identify models whose spectra most closely match the observed spectrum, the matched models are ideal starting models for carrying out inversions or more refined analyses. The method does require the computation of a large number of models; hence, it may not be the optimum method to use when studying a more extensive parameter set. Here the Brown et al. (1994) method could be used to complement the mode-matching method. Once the mode-matching method narrows down the possible set of model parameters in mass, age, and composition, the Brown et al. method could be used to expand the set of parameters.

The mode-matching method can immediately show whether or not the oscillation data are consistent with conventional stellar models, and it can show how well the oscillation data constrain the age, mass, and composition of conventional models. When a more extensive grid of models is completed, the mode-matching software can be used by anyone as a first line of investigation into interpreting their stellar oscillation data.

This work was supported in part by an NSERC grant to D. B. G. The computations were carried out on Saint Mary's University's High Performance Computer, a component of the Institute for Computational Astrophysics. D. B. G. would like to thank Gilles Fontaine, whose work along similar lines, but applied to the oscillation spectra of white dwarfs, inspired this investigation. D. B. G. would also like to thank Pierre Demarque for his helpful comments throughout this research. 
Alexander, D. R., \& Ferguson, J. W. 1994, ApJ, 437, 879

Appourchaux, T., et al. 1998, in Structure and Dynamics of the Interior of the Sun and Sun-like Stars, Proc. SOHO 6/GONG 98 Workshop, ed. S. Korzennik \& A. Wilson (Noordwijk: ESA), 99

Baglin, A., et al. 2002, in Proc. First Eddington Workshop on Stellar Structure and Habitable Planet Finding, ed. B. Battrick et al. (ESA SP485; Noordwijk: ESA), 17

Bahcall, J. N., Pinsonneault, M. H., \& Basu, S. 2001, ApJ, 555, 990

Bahcall, J. N., Pinsonneault, M. H., \& Wasserburg, G. J. 1995, Rev. Mod. Phys., 67, 781

Barban, C., Michel, E., Martic, M., Schmitt, J., Lebrun, J. C., Baglin, A., \& Bertaux, J. L. 1999, A\&A, 350, 617

Basu, S. 1997, MNRAS, 288, 572

Bouchy, F., \& Carrier, F. 2002, A\&A, 390, 205

Brown, T. M., Christensen-Dalsgaard, J., Weibel-Mihalas, B., \& Gilliland, R. L. 1994, ApJ, 427, 1013

Buzasi, D., Catanzarite, J., Laher, R., Conrow, T., Shupe, D., Gautier, T. N., III, Kreidl, T., \& Everett, D. 2000, ApJ, 532, L133

Canuto, V. M., \& Mazzitelli, I. 1991, ApJ, 370, 295 1992, ApJ, 389, 724

Chaboyer, B., Demarque, P., \& Guenther, D. B. 1999, ApJ, 525, L41

Chandrasekhar, S. 1957, An Introduction to the Study of Stellar Structure (New York: Dover)

Chaplin, W. J., Elsworth, Y., Isaak, G. R., Miller, B. A., \& New, R. 1999 , MNRAS, 308, 424

Christensen-Dalsgaard, J. 1986, in Seismology of the Sun and the Distant Stars, ed. D. O. Gough (Dordrecht: Reidel), 23

Christensen-Dalsgaard, J., Bedding, T. R., \& Kjeldsen, H. 1995, ApJ, 443, L29

Christensen-Dalsgaard, J., Gough, D. O., \& Thompson, M. J. 1991, ApJ, 378,413

Di Mauro, M. P., \& Christensen-Dalsgaard, J. 2001, in Helio- and Astroseimology at the Dawn of the Millennium, Proc. SOHO 10 GONG 2000 Workshop, ed. A. Wilson (Noordwijk: ESA), 373

Dziembowski, W. A., Gough, D. O., Houdek, G., \& Sienkiewicz, R. 2001, MNRAS, 328, 601

Dziembowski, W. A., Pamiatnykh, A. A., \& Sienkiewicz, R. 1991, MNRAS, 249, 602

Edmonds, P., Cram, L., Demarque, P., Guenther, D. B., \& Pinsonneault, M. H. 1992, ApJ, 394, 313

Favata, F., Roxburgh, I., \& Christensen-Dalsgaard, J. 2000, in The Third MONS Workshop: Science Preparation and Target Selection, ed. T. C. Teixeira \& T. R. Bedding (Copenhagen: Aarhus Univ.), 49

\section{EFERENCES}

Grevesse, N., Noels, A., \& Sauval, A. J. 1996, in ASP Conf. Ser. 99, Cosmic Abundances, ed. S. S. Holt \& G. Sonneborn (San Francisco: ASP), 117

Guenther, D. B. 1989, ApJ, 339, 1156

1994, ApJ, 422, 400

2002, ApJ, 569, 911

Guenther, D. B., \& Demarque, P. 1996, ApJ, 456, 798

1997, ApJ, 484, 937

2000, ApJ, 531, 503

Guenther, D. B., Demarque, P., Buzasi, D., Catanzarite, J., Laher, R., Conrow, T., \& Kreidl, T. 2000, ApJ, 530, L45

Guenther, D. B., Demarque, P., Kim, Y. -C., \& Pinsonneault, M. H. 1992, ApJ, 387, 372

Harvey, J. W., et al. 1996, Science, 272, 1284

Iglesias, C. A., \& Rogers, F. J. 1996, ApJ, 464, 943

Kjeldsen, H., \& Bedding T. R. 1995, A\&A, 293, 87

. 2001, in Helio- and Astroseimology at the Dawn of the Millennium, Proc. SOHO 10/GONG 2000 Workshop, ed. A. Wilson (Noordwijk: ESA), 361

Li, L. H., Robinson, F. J., Demarque, P., Sofia, S., \& Guenther, D. B. 2002, ApJ, 567, 1192

Matthews, J. M., et al. 2000, in IAU Colloq. 176, The Impact of LargeScale Surveys on Pulsating Star Research, ed. L. Szabados \& D. Kurtz (ASP Conf. Ser. 203; San Francisco: ASP), 74

Morel, P., Provost, J., Lebreton, Y., Thévenin, F., \& Berthomieu, G. 2000, A\&A, 363, 675

Robinson, F., Demarque, P., Li, L. H., Sofia, S., Kim, Y.-C., Chan, K. L., \& Guenther, D. B. 2003, MNRAS, 340, 923

Rogers, F. J. 1986, ApJ, 310, 723

Rogers, F. J., Swenson, F. J., \& Iglesias, C. A. 1996, ApJ, 456, 902

Rosenthal, C. S., Christensen-Dalsgaard, J., Nordlund, A ., Stein, R. F., \& Trampedach, R. 1999, A\&A, 351, 689

Schou, J., et al. 2002, ApJ, 567, 1234

Söderhjelm, S. 1999, A\&A, 341, 121

Tassoul, M. 1980, ApJS, 43, 469

Taylor, B. J. 1999, A\&AS, 134, 523

Thévenin, F., Provost, J., Morel, P., Berthomieu, G., Bouchy, F., \& Carrier, F. 2002, A\&A, 392, L9 